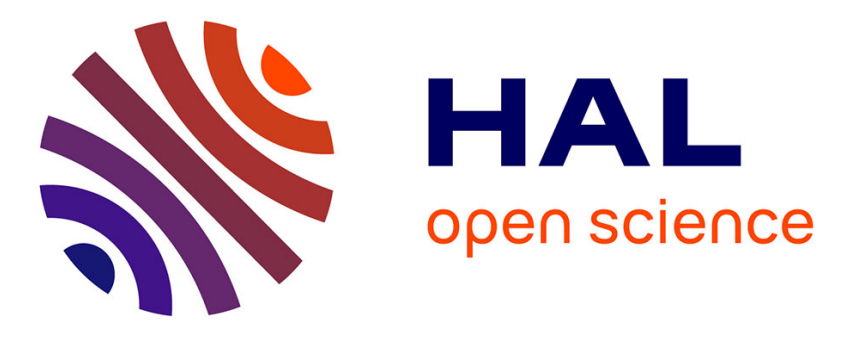

\title{
Relay Selection in Network-Coded Cooperative MIMO Systems
}

Ali Reza Heidarpour, Masoud Ardakani, Chintha Tellambura, Marco Di Renzo

\section{To cite this version:}

Ali Reza Heidarpour, Masoud Ardakani, Chintha Tellambura, Marco Di Renzo. Relay Selection in Network-Coded Cooperative MIMO Systems. IEEE Transactions on Communications, 2019, 67 (8), pp.5346-5361. 10.1109/TCOMM.2019.2911276 . hal-02395793

\section{HAL Id: hal-02395793 https://hal.science/hal-02395793}

Submitted on 6 Jul 2020

HAL is a multi-disciplinary open access archive for the deposit and dissemination of scientific research documents, whether they are published or not. The documents may come from teaching and research institutions in France or abroad, or from public or private research centers.
L'archive ouverte pluridisciplinaire HAL, est destinée au dépôt et à la diffusion de documents scientifiques de niveau recherche, publiés ou non, émanant des établissements d'enseignement et de recherche français ou étrangers, des laboratoires publics ou privés. 


\title{
Relay Selection in Network-Coded Cooperative MIMO Systems
}

\author{
Ali Reza Heidarpour, Student Member, IEEE, Masoud Ardakani, Senior Member, IEEE, Chintha \\ Tellambura, Fellow, IEEE, and Marco Di Renzo, Senior Member, IEEE
}

\begin{abstract}
Network-coded cooperation (NCC) has recently gained interest as it improves the network throughput in multisource cooperative systems. NCC has been studied for singleantenna terminals only. Employing multiple-input multipleoutput (MIMO) techniques can significantly improve the performance of NCC systems. Furthermore, the existing relay selection (RS) strategies for NCC utilize the "max-min" end-toend $($ E2E) criterion. This selection strategy (called Strategy $\mathcal{A}$ ) is complicated even for a network with single-antenna terminals as it requires global channel state information (CSI). This requirement makes it hard to implement RS-based NCC. To counter this issue, we introduce a new RS strategy (Strategy $\mathcal{B}$ ), which utilizes only the local CSI (not global CSI), reducing the signaling overhead significantly without sacrificing the performance. The performance of MIMO-NCC under Strategy $\mathcal{A}$ and $\mathcal{B}$ is studied over independent and non-identically distributed (i.n.i.d.) Rayleigh fading channels. Relays and the destination are equipped with multiple antennas, whereas sources have single antenna. The exact outage probability expressions of the system under consideration are derived. The asymptotic outage expressions are further provided to obtain valuable insights into the practical system-design parameters such as the diversity order and coding gain. Furthermore, numerical results are provided in support of the analytical results.
\end{abstract}

Keywords-Network-coded cooperation (NCC), multiple-input multiple-output (MIMO), relay selection (RS), outage probability, diversity order, coding gain.

\section{INTRODUCTION}

$\mathbf{M}$ ULTIPATH induced fading, which results in random fluctuation in the received signal level, is one of the major causes of the performance degradation in wireless networks. This degradation is commonly combated by using diversity techniques in time, frequency, and space. Distributed spatial diversity is exploited via cooperative communication (CC) [2]. CC makes use of the broadcast nature of the wireless channels and forms virtual antenna arrays among the nodes. Thus, relaying technologies have been widely adopted in broadband mobile standards such as 3GPP LTE-Advanced, IEEE 802.16j, and IEEE 802.16m [3].

In general, $\mathrm{CC}$ systems require two phases to transmit a message from the source to the destination: i) the broadcasting phase; and ii) the relaying phase. During the former, the source

This paper was presented in part at the IEEE 86th Vehicular Technology Conference, September 2017 [1]. This work was supported in part by the TELUS Corporation and Natural Sciences and Engineering Research Council of Canada.

A. R. Heidarpour, M. Ardakani, and C. Tellambura are with the Department of Electrical and Computer Engineering, University of Alberta, Edmonton, AB, T6G 1H9, Canada (e-mail: \{alirezaheidarpour, ardakani, ct4\}@ualberta.ca).

M. Di Renzo is with the L2S department, CNRS, CentraleSupelec, University of Paris-Sud, Universite Paris-Saclay, 91192 Gif-sur-Yvette, France (e-mail: marco.direnzo@12s.centralesupelec.fr). transmits its information, while the relays and the destination listen. During the latter, one or multiple relay(s) transmit(s) the received signal to the destinations after proper processing. In conventional multi-source multi-relay CC systems, however, each relay transmission must be coupled with a source transmission. Thus, each relay utilizes multiple resource blocks when forwarding messages for different sources. This timeslot usage results in throughput loss and becomes a major performance limiting factor for multi-source cooperative networks.

Inspired by the seminal work on network coding (NC) for wired networks [4], the joint use of CC and NC (which is referred to as network-coded cooperation (NCC)) has been proposed to improve the spectral efficiency of CC systems [5]-[7]. The main idea is that the relay node invokes NC by linearly combining data packets received from multiple sources, and then forwards the resulting signal to the destination. The time-resource allocation for an $N$-source, $M$ relay cooperative network varies depending on CC or NCC. In CC, each message of a single source is transmitted in $M+1$ time-slots. Thus, a total number of $N(M+1)$ time-slots are required. On the other hand, in NCC, during the broadcasting phase, $N$ sources transmit their information to the destination and the relays in $N$ orthogonal time-slots. During the relaying phase, each relay linearly combines (network coding) the received packets from the $N$ sources and then forwards the resulting network-coded packet to the destination in a single time-slot. As a result, only $N+M$ time-slots are required, which is much smaller than $N(M+1)$ time-slots for CC. Since NCC reduces the total transmission time, network throughput is significantly increased.

\section{A. Related Works}

NCC has been the subject of many recent studies [8]-[15]. In particular, in [8] the authors investigate network codes design for general $N$-source, $M$-relay wireless networks with a single destination, where codes are constructed in $q$-ary Galois field $\mathcal{G} \mathcal{F}(q) \mathrm{NC}$ and relays use decode-and-forward (DF) protocol. Their results reveal that binary $\mathrm{NC}$ based on the arithmetic $\mathcal{G F}(2)$ is not optimal to achieve full diversity order in a cooperative network with $M>1$. Instead, a non-binary $\mathrm{NC}$ based on maximum distance separable (MDS) codes is shown to provide the full diversity order of $M+1$ for any arbitrary $M$ and $N$. Furthermore, the diversity order of $M-N+1$ can be achieved if direct source-to-destination channels are not available, which is equivalent to achieving Singleton bound in error correction codes. The outage probability and diversitymultiplexing tradeoff (DMT) of NCC based on DF relaying has been further studied in [9], showing that NCC is capable 
of achieving full diversity order and outperforms conventional CC systems in terms of DMT. These works have triggered other research efforts to investigate the performance of NCC systems for various system models (see e.g., [11]-[15]).

Since NCC systems use multiple orthogonal channels, relay selection (RS) protocols can further improve the spectral efficiency of NCC systems. Few sporadic works investigate the performance of RS NCC systems [16]-[19]. More specifically, in [16], the performance of the XOR-based NCC with RS for multiple source-destination pairs is analyzed. Later, $\mathrm{Vu}$ et al. investigated RS for non-binary NCC with two RS protocols, namely single RS (SRS) and multiple RS (MRS) [18]. Their results demonstrate that as opposed to conventional CC systems in which SRS achieves full diversity order [20], SRS for NCC systems achieves a maximum diversity order of two irrespective of the number of sources and relays. Furthermore, MRS can provide full diversity order, depending on the number of selected relays.

\section{B. Motivations and Contributions}

Despite the rich literature on NCC, all existing works [8]-[19] are restricted to relay networks with single-antenna terminals. Employing multiple antennas at the transmitter and/or receiver has been identified as a key enabling technique for future generation of wireless networks and has been broadly investigated in the context of CC systems [21]-[24]. The applications of MIMO techniques on NCC networks are also interesting, which have been lacking in the literature. Furthermore, not only MIMO-NCC is not studied in the literature, but also the existing NCC RS strategies rely on the "max-min" end-to-end (E2E) criterion. This selection strategy (called Strategy $\mathcal{A}$ ) will be too complicated even for a network with single-antenna terminals as it requires global channel state information (CSI). Such high signaling overhead leads to difficult implementation of NCC system with RS, especially for a network with large number of branches. It is thus important to devise efficient RS schemes with limited overhead. One of the key contributions of our work here is to propose and analyze a new RS strategy (Strategy $\mathcal{B}$ ) based on the local CSI of the relay-to-destination channels (rather than global CSI), resulting in a significantly reduced signaling overhead. Motivated by these key observations, this paper presents the first comprehensive performance analysis of MIMO-NCC systems with the two different RS strategies.

In particular, we consider a practical scenario where $N>1$ single-antenna sources communicate with a multiple-antenna destination using $M \geq 1$ multiple-antenna relays. This scenario could be an instance of the cellular uplink, where single-antenna mobile terminals communicate with a multipleantenna base station with the help of infrastructure relays. ${ }^{1}$ This is a practical assumption, since use of multiple antennas at the relays and the base station is reasonable, while the use of multiple antennas at mobile terminals are restricted due to the size constraint, transmission power, and circuit complexity.

\footnotetext{
${ }^{1}$ Infrastructure relays are applicable in practice to infrastructure-based relay networks, where relays are fixed and therefore can be equipped with multiple antennas [25].
}

Among various strategies to exploit multiple antennas, we consider transmit antenna selection (TAS) at the transmitter side. This choice is made because TAS is easy to implement with low feedback signaling [26]. On the other hand, maximal-ratio combining (MRC) at the receiver side is able to maximize the diversity order and the received signal-to-noise ratio (SNR). Therefore, in our system setup, the relays and destination use MRC for signal reception and relays use TAS for forwarding the encoded signals to the destination.

The contributions of this work can be summarized as follows: (i) Attracted by the benefits of multi-antenna techniques in enhancing NCC system performance, we extend single-antenna NCC to a multi-antenna scenario. (ii) A new RS strategy (Strategy $\mathcal{B}$ ) for NCC systems is proposed and analyzed. It can substantially reduce the required signaling overhead for RS-based NCC, particularly in a network with large number of branches without sacrificing the performance. RS Strategy $\mathcal{B}$ is different from those based on "max-min" criterion [17]-[19] because it requires local CSI only. Therefore, our analytical analysis are new and completely different from those earlier reported in [17]-[19]. (iii) We derive exact outage probability expressions for MIMO-NCC system with RS strategies $\mathcal{A}, \mathcal{B}$ over independent and non-identically distributed (i.n.i.d.) Rayleigh fading channels. (iv) The asymptotic outage expressions are further provided to characterize the key performance indicators such as the diversity order and coding gain. (v) We further present special cases for our derived analytical expressions to demonstrate that they are generalized versions of those earlier presented in the literature and coincide with them when single-antenna terminals are considered. (vi) We compare the outage performance of the proposed RS MIMO-NCC with two benchmark schemes, namely singleantenna RS NCC [17], [18], and RS MIMO-NCC with random antenna selection (RAS) at relays. (vii) Numerical results are also presented to validate the accuracy of our derivations and quantify the effect of different system parameters on the outage probability and diversity order.

\section{Structure and Notations}

The remainder of this paper is organized as follows. In Section II, the system model under consideration is described. In Section III, we provide some basic definitions and preliminaries. In Section IV, the outage probability and asymptotic expressions of MIMO-NCC with RS Strategy $\mathcal{A}$ are derived. Section V provides the performance of MIMO-NCC with RS Strategy $\mathcal{B}$. Analytical results and Monte-Carlo simulations are compared in Section VI. Finally, Section VII concludes this paper.

Notations: In this paper, $\operatorname{Pr}\{\cdot\}, \mathcal{C}_{m}^{n}=\frac{n !}{(n-m) ! m !}$, and $\|\cdot\|$ denote probability, binomial coefficient, and norm of a vector, respectively. $F_{X}(\cdot)$ denotes a cumulative distribution function $(\mathrm{CDF})$ for a random variable $X . \mathcal{C N}\left(\mu, \sigma^{2}\right)$ denotes a circularly symmetric complex Gaussian random variable with mean $\mu$ and variance $\sigma^{2}$.

\section{SySTEM MODEL}

We consider a MIMO-NCC system consisting of $N$ sources $S_{n}, n \in\{1,2, \ldots, N\}, M$ relays $R_{m}, m \in\{1,2, \ldots, M\}$, 
and one destination $D$. In particular, sources have single antenna, whereas relays and the destination are equipped with $N_{r} \geq 1$ and $N_{d} \geq 1$ antennas, respectively. All terminals operate in half-duplex mode. We assume flat fading uncorrelated Rayleigh fading channels, where $\mathbf{h}_{S_{n} R_{m}} \in \mathbb{C}^{N_{r} \times 1}$, $\mathbf{h}_{S_{n} D} \in \mathbb{C}^{N_{d} \times 1}$, and $\mathbf{H}_{R_{m} D} \in \mathbb{C}^{N_{d} \times N_{r}}$, respectively denote the channel for $S_{n} \rightarrow R_{m}, S_{n} \rightarrow D$, and $R_{m} \rightarrow D$ links. The $N_{d} \times 1$ channel vector of the $i^{t h}$ transmit antenna for $R_{m} \rightarrow D$ is denoted by $\mathbf{h}_{R_{m}^{(i)} D}$. We make the practical assumption of i.n.i.d. Rayleigh fading channels, where the signals transmitted through different links experience different radio environments. In particular, the elements of $\mathbf{h}_{S_{n} R_{m}}$, $\mathbf{h}_{S_{n} D}$, and $\mathbf{H}_{R_{m} D}$ are modeled as $\mathcal{C N}\left(0, \lambda_{n, m}\right), \mathcal{C N}\left(0, \lambda_{n}\right)$, and $\mathcal{C N}\left(0, \lambda_{m}\right)$. Furthermore, the noise corresponding to each channel is independent additive white Gaussian noise (AWGN) with $\mathcal{C N}\left(0, N_{0}\right)$.

In our system, cooperation takes place in two phases, namely the broadcasting and relaying phases. During the broadcasting phase, the sources transmit their symbols (each consisting of $\chi$ bits) to the destination in $N$ non-overlapping time slots. Both the destination and relays employ MRC reception to decode the data symbols received from the sources. During the relaying phase, sources remain silent and the selected relay(s) (see Sections IV and $\mathrm{V}$ for more details) apply(s) $\mathrm{NC}$ in $\mathcal{G} \mathcal{F}\left(2^{\chi}\right)$ and then forward(s) networkcoded symbols to the destination using TAS. The detailed information about how the relays combine sources' messages using NC can be found in [9], [17]. Finally, the destination decodes encoded symbols received from the relays in the relaying phase by using MRC. We assume a centralized RS method where the RS process, for both Strategy $\mathcal{A}$ and $\mathcal{B}$, is performed by a central unit (e.g, the destination). An instance of this system could be the cellular uplink, where $N>1$ single-antenna mobile terminals communicate with a multipleantenna base station with the help of $M \geq 1$ multiple-antenna relays.

\section{BASIC DEFINITIONS AND PRELIMINARIES}

Basic definitions of system-design parameters such as the diversity order and coding gain are provided. The outage probability expressions of single-hop links are further derived to facilitate the overall outage analysis of MIMO-NCC system.

\section{A. Diversity Order and Coding Gain}

At high SNR regime, the overall outage probability is approximated as

$$
\mathcal{P}_{\text {out }}^{\infty} \stackrel{\bar{\gamma}}{\stackrel{\sim}{\approx}}\left(G_{c} \bar{\gamma}\right)^{-G_{d}}
$$

In (1), $\bar{\gamma}$ is the SNR. Furthermore, $G_{c}$ and $G_{d}$ are respectively, called as "diversity order", and "coding gain". In particular, $G_{c}$ is the horizontal shift in the outage cure with respect to the benchmark curve $(\bar{\gamma})^{-G_{d}}$ and $G_{d}$ is defined as the slope of the asymptotic outage curve and is given by

$$
G_{d}=\lim _{\bar{\gamma} \rightarrow \infty}-\frac{\log \left(\mathcal{P}_{\text {out }}\right)}{\log (\bar{\gamma})} .
$$

\section{B. Outage Probability of Single-Hop Links}

In NCC systems, the outage probabilities of the source-torelay, source-to-destination, and relay-to-destination links are the building blocks of overall outage probability of the system. Therefore, the outage probability calculation for each singlehop link is required to evaluate the E2E performance.

The single-hop link $X \rightarrow Y$ is in outage if it cannot support the fixed transmission rate $\mathcal{R}_{0}$ (in bits per channel use). The corresponding outage probability is given by

$$
\mathcal{P}_{O_{X Y}}\left(\mathcal{R}_{0}\right)=\operatorname{Pr}\left\{\mathcal{I}\left(\gamma_{X Y}\right)<\mathcal{R}_{0}\right\},
$$

where $\mathcal{I}\left(\gamma_{X Y}\right)$ is the instantaneous mutual information corresponding to the equivalent received SNR $\gamma_{X Y}$. Noting that $\mathcal{I}\left(\gamma_{X Y}\right)=\log _{2}\left(1+\gamma_{X Y}\right)$, (3) can be rewritten as

$$
\mathcal{P}_{o_{X Y}}=\operatorname{Pr}\left\{\gamma_{X Y}<\gamma_{t h}\right\}=F_{\gamma_{X Y}}\left(\gamma_{t h}\right),
$$

where $\gamma_{t h}=2^{\mathcal{R}_{0}}-1$ is the threshold SNR.

Since relays and the destination employ MRC at the receiver side, the equivalent instantaneous SNR for $S_{n} \rightarrow R_{m}$ and $S_{n} \rightarrow D$ links are respectively given by $\gamma_{n, m}=\bar{\gamma}\left\|\mathbf{h}_{S_{n} R_{m}}\right\|^{2}$, and $\gamma_{n}=\bar{\gamma}\left\|\mathbf{h}_{S_{n} D}\right\|^{2}$, where $\bar{\gamma}$ is the transmit SNR. Similarly, for the $i^{\text {th }}$ transmit antenna at the relay $R_{m}$ in the relaying phase, the equivalent instantaneous SNR is given by $\gamma_{m}^{(i)}=\bar{\gamma}\left\|\mathbf{h}_{R_{m}^{(i)} D}\right\|^{2}$. Furthermore, $\gamma_{n, m}, \gamma_{n}$, and $\gamma_{m}^{(i)}$ are independent Gamma distributed random variables with $\gamma_{n, m} \sim$ $\mathcal{G}\left(N_{r}, \bar{\gamma}_{n, m}\right), \gamma_{n} \sim \mathcal{G}\left(N_{d}, \bar{\gamma}_{n}\right)$, and $\gamma_{m}^{(i)} \sim \mathcal{G}\left(N_{d}, \bar{\gamma}_{m}\right)$, where $\bar{\gamma}_{n, m}=\bar{\gamma} \lambda_{n, m}, \bar{\gamma}_{n}=\bar{\gamma} \lambda_{n}$, and $\bar{\gamma}_{m}=\bar{\gamma} \lambda_{m}$. Replacing $\gamma_{n, m}$, and $\gamma_{n}$ in (4), the outage probability of $S_{n} \rightarrow R_{m}$ and $S_{n} \rightarrow D$ links are respectively, given by

$$
\begin{gathered}
F_{\gamma_{n, m}}\left(\gamma_{t h}\right)=1-e^{-\frac{\gamma_{t h}}{\bar{\gamma}_{n, m}}} \sum_{i=1}^{N_{r}} \frac{\left(\gamma_{t h} / \bar{\gamma}_{n, m}\right)^{i-1}}{(i-1) !}, \\
F_{\gamma_{n}}\left(\gamma_{t h}\right)=1-e^{-\frac{\gamma_{t h}}{\bar{\gamma}_{n}}} \sum_{j=1}^{N_{d}} \frac{\left(\gamma_{t h} / \bar{\gamma}_{n}\right)^{j-1}}{(j-1) !} .
\end{gathered}
$$

Furthermore, the outage probability of the channel between the $i^{\text {th }}$ transmit antenna at $R_{m}$ and $D$ is given by

$$
F_{\gamma_{m}^{(i)}}\left(\gamma_{t h}\right)=1-e^{-\frac{\gamma_{t h}}{\bar{\gamma}_{m}}} \sum_{j=1}^{N_{d}} \frac{\left(\gamma_{t h} / \bar{\gamma}_{m}\right)^{j-1}}{(j-1) !} \text {. }
$$

Note that $\bar{\gamma}_{n, m}, \bar{\gamma}_{n}$, and $\bar{\gamma}_{m}$ in (5), (6), and (7), respectively denote the average received SNR of $S_{n} \rightarrow R_{m}, S_{n} \rightarrow D$, and $R_{m} \rightarrow D$ links, including path-loss.

\section{Discussion}

In RS Strategy $\mathcal{B}$ the destination selects the best relays based on the local CSI of the relay-to-destination links. The RS Strategy $\mathcal{A}$, however, relies on global CSI; i.e., not only the destination requires local CSI of the relay-to-destination links but also it requires CSI of the indirectly connected source-to-relay links. Thus, as opposed to $\mathrm{RS}$ Strategy $\mathcal{B}$, in RS Strategy $\mathcal{A}$ the relays need to send the CSI information to the destination. Suppose relay $R_{m}(\forall m \in\{1,2, \ldots, M\})$ obtains CSI of its corresponding source-to-relay links using pilot sequences sent by $N$ sources. Furthermore, assume that real and imaginary parts are quantized with $L$ bits each, and a 
rate $\mathcal{C}$ channel code is employed to protect the CSI of sourceto-relay channels. Noting that MRC is employed at relays and the resulting combined SNRs are used for RS process, the total number of bits needed for CSI acquisition at the destination in Strategy $\mathcal{A}$ is $\mathcal{T}_{b}^{\mathcal{A}}=\frac{2 L N M}{\mathcal{C}}$, where the factor of two is due to the complex component. On the other hand, the number of required CSI estimations in RS Strategy $\mathcal{A}$ and $\mathcal{B}$ are respectively equal to $\mathcal{N}_{\mathcal{A}}=M(N+1)$ and $\mathcal{N}_{\mathcal{B}}=M$. It can be seen that the additional signaling overhead imposed by RS Strategy $\mathcal{A}$ is scaled by the product of the number of sources $N$ and number of relays $M$. Such high signaling overhead is even more crucial in a network with large number of branches (i.e., $N \gg 1, M \gg 1$ ). This additional signaling overhead clearly demonstrates the superiority of RS Strategy $\mathcal{B}$ over RS Strategy $\mathcal{A}$.

In the next two sections, we explain RS Strategy $\mathcal{A}, \mathcal{B}$ in detail and derive their outage probabilities.

\section{RS STRATEGY $\mathcal{A}$}

In RS under Strategy $\mathcal{A}, K$ relays (out of $M$ cooperative relays) based on "max-min" criterion are selected to maximize the worst E2E SNR. According to "max-min" criterion, the E2E performance is dominated by the worst link between $S_{n} \rightarrow R_{m},(n=1,2, \ldots, N)$ and $R_{m} \rightarrow D$ links. In other words, amongst the links corresponding to relay $R_{m}$, the link whose instantaneous SNR is less than that of others determines the bottleneck link. Let $\gamma_{m}^{b}$ denote the SNR of the bottleneck link for relay $R_{m}$. Accordingly, based on "maxmin" criterion, relays $R_{(1)}, R_{(2)}, \cdots, R_{(K)}$ are selected, where $(v)=\operatorname{argmax}_{m=1,2, \cdots, M}^{v \text { th }}\left\{\gamma_{m}^{b}\right\}$. During the relaying phase, these $K$ best relays participate in a round-robin fashion. In particular, in the first relaying time-slot, the best relay $R_{(1)}$ transmits; then, in the second relaying time-slot, the second best relay $R_{(2)}$ transmits; and this procedure continues until relay $R_{(K)}$ transmits. $^{2}$

\section{A. Outage Probability}

In this section, we derive closed-form outage expression for MIMO-NCC systems with RS Strategy $\mathcal{A}$. The high SNR approximation of the outage probability is also provided to obtain valuable insights into the system-design parameters such as the diversity order and coding gain.

Since, MRC is employed at the relay $R_{m}$ and a single best antenna $\hat{i}$ at this relay is selected to transmit encoded symbols in the relaying phase, the SNR of the bottleneck link for relay $R_{m}$ can be written as [16]-[19]

$$
\gamma_{m}^{b}=\min \left\{\gamma_{1, m}, \gamma_{2, m}, \ldots, \gamma_{N, m}, \gamma_{m}^{(\hat{i})}\right\}
$$

where $\gamma_{m}^{(\hat{i})}=\max _{i=1,2, \ldots, N_{r}} \bar{\gamma}_{m}\left\|\mathbf{h}_{R_{m}^{(i)} D}\right\|^{2}$ is the maximum SNR value among all the output instantaneous SNRs of the MRC at the destination.

\footnotetext{
${ }^{2}$ In the RS Strategy $\mathcal{A}$, the relays apply $\mathrm{NC}$ on received symbols that are either correctly or incorrectly demodulated. This implies that the RS Strategy $\mathcal{A}$ is affected by the error propagation effect. Our analysis assumes that the error propagation is counteracted at the destination with the aid of appropriate "error-aware" demodulators that are capable of providing fulldiversity. Further information is available in [17].
}

Using (8), the outage probability of relay $R_{m}$ can be then expressed as

$$
F_{\gamma_{m}^{b}}\left(\gamma_{t h}\right)=1-\operatorname{Pr}\left\{\gamma_{m}^{b}>\gamma_{t h}\right\} .
$$

Noting that the SNRs in (8) are mutually independent random variables, and using the theory of order statistics, (9) can be written as

$$
F_{\gamma_{m}^{b}}\left(\gamma_{t h}\right)=1-\prod_{n=1}^{N}\left[1-F_{\gamma_{n, m}}\left(\gamma_{t h}\right)\right]\left[1-F_{\gamma_{m}^{(\hat{i})}}\left(\gamma_{t h}\right)\right] .
$$

Applying the following multinomial expansion identity in (10)

$$
\prod_{\ell=1}^{L}\left(1-x_{\ell}\right)=1+\sum_{k=1}^{L}(-1)^{k} \sum_{\substack{i_{1}=1, \cdots, i_{k}=1 \\ i_{1}<\cdots<i_{k}}}^{L} \prod_{m=i_{1}}^{i_{k}} x_{m},
$$

we obtain

$$
\begin{aligned}
& \prod_{n=1}^{N}\left[1-F_{\gamma_{n, m}}\left(\gamma_{t h}\right)\right]=1+\sum_{k=1}^{N}(-1)^{k} \sum_{\substack{i_{1}=1, \cdots, i_{k}=1 \\
i_{1}<\cdots<i_{k}}}^{N} \\
& \prod_{n=i_{1}}^{i_{k}} F_{\gamma_{n, m}}\left(\gamma_{t h}\right)
\end{aligned}
$$

where $i_{1}, i_{2}, \cdots, i_{N} \in\{1,2, \cdots, N\}$.

On the other hand, $F_{\gamma_{m}^{(\hat{i})}}\left(\gamma_{t h}\right)$ in (10) is given by

$$
F_{\gamma_{m}^{(\hat{i})}}\left(\gamma_{t h}\right)=\left(1-e^{-\frac{\gamma_{t h}}{\bar{\gamma} m}} \sum_{j=1}^{N_{d}} \frac{\left(\gamma_{t h} / \bar{\gamma}_{m}\right)^{j-1}}{(j-1) !}\right)^{N_{r}}
$$

Let $\gamma_{(1)}^{b} \geq \gamma_{(2)}^{b} \geq \cdots \geq \gamma_{(M)}^{b}$ denote the order statistics of bottleneck SNRs of the relays in a decreasing order of magnitude, where $\gamma_{(v)}^{b}=\max _{m=1,2, \cdots, M}^{v \text { th }}\left\{\gamma_{m}^{b}\right\}$ is the $v$ th largest SNR. Then, we can write

$$
\begin{aligned}
\operatorname{Pr}\left\{\gamma_{(v)}^{b}<\gamma, \gamma_{(v-1)}^{b}>\gamma\right\} & =\operatorname{Pr}\left\{\left(v-1 \text { of } \gamma_{m}^{b} \text { 's }>\gamma\right)\right. \\
& \left.\bigcap\left(M-v+1 \text { of } \gamma_{m}^{b} \text { 's }<\gamma\right)\right\} .
\end{aligned}
$$

Since $\gamma_{m}^{b}$ 's are mutually independent random variables, (14) can be expresses as

$$
F_{\gamma_{(v)}^{b}}(\gamma)=\sum_{i_{1}, \cdots, i_{M}}^{M} \prod_{m=i_{1}}^{i_{v-1}}\left[1-F_{\gamma_{m}^{b}}(\gamma)\right] \prod_{m^{\prime}=i_{v}}^{i_{M}} F_{\gamma_{m^{\prime}}^{b}}(\gamma)
$$

where $i_{1}, i_{2}, \cdots, i_{M} \in\{1,2, \cdots, M\}, i_{1} \neq i_{2} \neq \cdots \neq i_{M}$, $i_{1}<i_{2}<\cdots<i_{v-1}$, and $i_{v}<i_{2}<\cdots<i_{M}$.

We can write (15) in a simple-form expression as

$$
F_{\gamma_{(v)}^{b}}(\gamma)=\sum_{k=1}^{v} \mathcal{C}_{M-v+1}^{M-v+k}(-1)^{k-1} \sum_{\substack{i_{1}, i_{2}, \cdots, i_{M-v+k} \\ i_{M-v+k}}}^{M} F_{\gamma_{m}^{b}}(\gamma) .
$$


Furthermore, the probability that $\tau$ sources be operational (i.e., not in outage) and the remaining $N-\tau$ sources be in outage in the broadcasting phase is given by

$$
\Phi(\tau)=\sum_{k=1}^{\tau+1} \mathcal{C}_{N-\tau}^{N+k-\tau-1}(-1)^{k-1} \sum_{\substack{i_{1}, i_{2}, \cdots, i_{N+k-\tau-1} \\ i_{N+k-\tau-1}}}^{N} F_{\gamma_{n}}\left(\gamma_{t h}\right) .
$$

In (17), $i_{1}, i_{2}, \cdots, i_{N} \in\{1,2, \cdots, N\}, i_{1}<i_{2}<\cdots<i_{N}$, and $F_{\gamma_{n}}\left(\gamma_{t h}\right)$ is given by (6). Furthermore, for the case of $i_{0}$ we have one.

In RS Strategy $\mathcal{A}$, the destination receives potentially $N+K$ packets; $N$ original packets from direct transmissions and $K$ network-coded packets from selected relays. An outage occurs if fewer than $N$ packets are received. Let $N_{\text {op }} \leq N$, and $K_{\text {op }}^{\mathcal{A}} \leq K$ denote the number of operational sources and relays, respectively. In general, there exist two different events which lead to the outage of the system and can be written as

$$
\mathcal{O}_{\mathcal{A}}=\mathcal{O}_{\mathcal{A}}^{(1)} \bigcup \mathcal{O}_{\mathcal{A}}^{(2)}
$$

where

(i) $\mathcal{O}_{\mathcal{A}}^{(1)}$ denotes the outage events when $N_{\text {op }}+K<N$, implying that there are not enough operational $S \rightarrow D$ links, $N_{\text {op }}$, such that even if all $K$ selected relays be operational i.e., $K_{\mathrm{op}}=K$, the system is still in outage. Note that $\mathcal{O}_{\mathcal{A}}^{(1)}$ only occurs when $N>K$.

(ii) $\mathcal{O}_{\mathcal{A}}^{(2)}$ represents the outage events when $N_{\text {op }}+K \geq N$ but $N_{\text {op }}+K_{\text {op }}^{\mathcal{A}}<N$.

From (18), the overall outage probability can be expressed as

$$
\operatorname{Pr}\left\{\mathcal{O}_{\mathcal{A}}\right\}=\operatorname{Pr}\left\{\mathcal{O}_{\mathcal{A}}^{(1)}\right\}+\operatorname{Pr}\left\{\mathcal{O}_{\mathcal{A}}^{(2)}\right\}
$$

Considering all outage events, the exact closed-form expressions for outage probability of MIMO-NCC system with RS Strategy $\mathcal{A}$ when $N>K$ can be derived as (20), where $F_{\gamma_{(v)}^{b}}(\gamma)$ and $\Phi(\tau)$ are already given by (16) and (17), respectively.

$$
\mathcal{P}_{\text {out }_{1}}^{\mathcal{A}}=\underbrace{\sum_{\tau=0}^{N-K-1} \Phi(\tau)}_{\operatorname{Pr}\left\{\mathcal{O}_{\mathcal{A}}^{(1)}\right\}}+\underbrace{\sum_{\tau=1}^{K}\left(\Phi(N-\tau) \sum_{v=1}^{\tau} F_{\gamma_{(v)}^{b}}\left(\gamma_{t h}\right)\right)}_{\operatorname{Pr}\left\{\mathcal{O}_{\mathcal{A}}^{(2)}\right\}} .
$$

On the other hand, the outage probability of the system when $N \leq K$ can be formulated as

$$
\mathcal{P}_{\text {out }_{2}}^{\mathcal{A}}=\underbrace{\sum_{\tau=1}^{N}\left(\Phi(N-\tau) \sum_{v=1}^{\tau} F_{\gamma_{(v)}^{b}}\left(\gamma_{t h}\right)\right)}_{\operatorname{Pr}\left\{\mathcal{O}_{\mathcal{A}}^{(2)}\right\}} .
$$

Although the derived outage probability expressions given by (20) and (21) are exact and valid for any arbitrary SNR values, direct insights into the effect of different system parameters on the outage performance are desirable. Motivated by this, we turn our attention to obtain the asymptotic outage expressions in high SNR regime which easily enable us to obtain the diversity order and coding gain.

\section{B. Asymptotic Analysis}

For asymptotically high SNR values, we express exponential function in terms of its Taylor series expansions given by $e^{-x}=\sum_{k=0}^{\infty} \frac{(-x)^{k}}{k !}$ to approximate (6) in high SNR regime. This is given by

$$
\lim _{\bar{\gamma} \rightarrow \infty} F_{\gamma_{n}}\left(\gamma_{t h}\right)=F_{\gamma_{n}}^{\infty}\left(\gamma_{t h}\right)=\frac{\beta_{n}^{N_{d}}}{N_{d} !}
$$

where $\beta_{n}=\gamma_{t h} / \bar{\gamma}_{n}$.

Substituting (22) into (17) and then ignoring higher order terms, we find

$$
\Phi^{\infty}(\tau)=\sum_{i_{1}, i_{2}, \cdots, i_{N-\tau}}^{N} \prod_{n=i_{1}}^{i_{N-\tau}} \frac{\beta_{n}^{N_{d}}}{N_{d} !} .
$$

Furthermore, we approximate $F_{\gamma_{m}^{b}}\left(\gamma_{t h}\right)$ in (10) as follows

$$
F_{\gamma_{m}^{b}}^{\infty}\left(\gamma_{t h}\right)=\eta+\sum_{i_{1}=1}^{N} \frac{\beta_{i_{1}, m}^{N_{r}}}{N_{r} !}
$$

In (24), $\beta_{n, m}=\gamma_{t h} / \bar{\gamma}_{n, m}$. Also, $\eta=0$ if $N_{d} \neq 1$ and $\eta=$ $\beta_{m}^{N_{r}}$ if $N_{d}=1$, where $\beta_{m}=\gamma_{t h} / \bar{\gamma}_{m}$.

Plugging (24) into (16) and then keeping the dominant terms, we obtain

$$
F_{\gamma_{(v)}^{b}}^{\infty}\left(\gamma_{t h}\right)=\sum_{i_{1}, i_{2}, \cdots, i_{M-v+1}}^{M} \prod_{m=i_{1}}^{i_{M-v+1}}\left(\eta+\sum_{i_{1}=1}^{N} \frac{\beta_{i_{1}, m}^{N_{r}}}{N_{r} !}\right) .
$$

Substituting (23) and (25) in (20), we have

$$
\mathcal{P}_{\text {out }_{1}^{\infty}}^{\mathcal{A}}=\sum_{\tau=0}^{N-K-1} \Phi^{\infty}(\tau)+\sum_{\tau=1}^{K}\left(\Phi^{\infty}(N-\tau) \sum_{v=1}^{\tau} F_{\gamma_{(v)}}^{\infty}\left(\gamma_{t h}\right)\right) .
$$

Keeping the dominant terms in (26) i.e., when $\tau=N-K-1$ in the first summation and $v=\tau=1$ in the second and third summations, (20) can be further approximated as

$$
\mathcal{P}_{\text {out }_{1}^{\infty}}^{\mathcal{A}}=\Phi^{\infty}(N-K-1)+\Phi^{\infty}(N-1) F_{\gamma_{(1)}}^{\infty}\left(\gamma_{t h}\right) .
$$

The asymptotic outage expression depends on the system parameters. In particular, we have the following three cases:

Case 1: $M N_{r}>K N_{d}$. In this case, $\mathcal{P}_{\text {out }_{1}^{\mathcal{A}}}^{\mathcal{A}}$ is determined by the first term in (27) as $\mathcal{P}_{\text {out }_{1}^{\infty}}^{\mathcal{A}}=\Phi^{\infty}(N-K-1)$ and is given by

$$
\mathcal{P}_{\text {out }_{1}^{\infty}}^{\mathcal{A}}=\Xi_{1}^{\mathcal{A}}\left(\frac{\gamma_{t h}}{\bar{\gamma}}\right)^{(K+1) N_{d}}
$$

where the system-dependent parameter, $\Xi_{1}^{\mathcal{A}}$, is

$$
\Xi_{1}^{\mathcal{A}}=\sum_{i_{1}, i_{2}, \cdots, i_{K+1}}^{N} \prod_{n=i_{1}}^{i_{K+1}} \frac{\lambda_{n}^{-N_{d}}}{N_{d} !}
$$

Case 2: $M N_{r}<K N_{d}$. In this case, $\mathcal{P}_{\text {out }_{1}^{\infty}}^{\mathcal{A}}$ is determined by the second term in (27) as $\mathcal{P}_{\text {out }_{1}^{\infty}}^{\mathcal{A}}=\Phi^{\infty}(N-1) F_{\gamma_{(1)}}^{\infty}\left(\gamma_{t h}\right)$. This can be written as

$$
\mathcal{P}_{\text {out }_{1}^{\infty}}^{\mathcal{A}}=\Xi_{1^{\prime}}^{\mathcal{A}}\left(\frac{\gamma_{t h}}{\bar{\gamma}}\right)^{M N_{r}+N_{d}},
$$


where

$$
\Xi_{1^{\prime}}^{\mathcal{A}}=\sum_{i_{1}=1}^{N} \frac{\lambda_{i_{1}}^{-N_{d}}}{N_{d} !} \prod_{m=i_{1}}^{i_{M}}\left(\sum_{i_{1}=1}^{N} \frac{\lambda_{i_{1}, m}^{-N_{r}}}{N_{r} !}\right) .
$$

Case 3: $M N_{r}=K N_{d}$. In this case, both the first and the second terms in (27) determine the asymptotic outage given by $\mathcal{P}_{\text {out }_{1}^{\infty}}^{\mathcal{A}}=\Phi^{\infty}(N-K-1)+\Phi^{\infty}(N-1) F_{\gamma_{(1)}}^{\infty}\left(\gamma_{t h}\right)$, where $\Phi^{\infty}\left(N^{1}-K-1\right)$, and $\Phi^{\infty}(N-1) F_{\gamma_{(1)}}^{\infty}\left(\gamma_{t h}\right)$ are derived in (28) and (30), respectively.

Now, we proceed to obtain the asymptotic outage expression for $N \leq K$. Substituting (23) and (25) into (21), we have

$$
\mathcal{P}_{\text {out }_{2}^{\infty}}^{\mathcal{A}}=\sum_{\tau=1}^{N}\left(\Phi^{\infty}(N-\tau) \sum_{v=1}^{\tau} F_{\gamma_{(v)}^{b}}^{\infty}\left(\gamma_{t h}\right)\right) .
$$

Based on the relationship between $N_{d}$ and $N_{r}$, (21) in high SNRs is derived in the following three cases:

Case 1: $N_{d}>N_{r}$. In this case, the dominant terms of (32) can be obtained when $v=\tau=1$ i.e., $\mathcal{P}_{\text {out }_{2}^{\infty}}^{\mathcal{A}}=\Phi^{\infty}(N-$ 1) $F_{\gamma_{(1)}}^{\infty}\left(\gamma_{t h}\right)$. This means

$$
\mathcal{P}_{\text {out }_{2}^{\infty}}^{\mathcal{A}}=\Xi_{2}^{\mathcal{A}}\left(\frac{\gamma_{t h}}{\bar{\gamma}}\right)^{M N_{r}+N_{d}}
$$

where

$$
\Xi_{2}^{\mathcal{A}}=\sum_{i_{1}=1}^{N} \frac{\lambda_{i_{1}}^{-N_{d}}}{N_{d} !} \prod_{m=i_{1}}^{i_{M}}\left(\sum_{i_{1}=1}^{N} \frac{\lambda_{i_{1}, m}^{-N_{r}}}{N_{r} !}\right) .
$$

Case 2: $N_{d}<N_{r}$. In this case, the dominant terms of (32) can be obtained when $\tau=v=N$ i.e., $\mathcal{P}_{\text {out }_{2}^{\infty}}^{\mathcal{A}}=$ $\Phi^{\infty}(0) F_{\gamma_{(N)}}^{\infty}\left(\gamma_{t h}\right)$. This can be expressed as

$$
\mathcal{P}_{\text {out }_{2}^{\infty}}^{\mathcal{A}}=\Xi_{2^{\prime}}^{\mathcal{A}}\left(\frac{\gamma_{t h}}{\bar{\gamma}}\right)^{(M-N+1) N_{r}+N N_{d}},
$$

where

$$
\Xi_{2^{\prime}}^{\mathcal{A}}=\prod_{n=i_{1}}^{i_{N}} \frac{\lambda_{n}^{-N_{d}}}{N_{d} !} \sum_{i_{1}, i_{2}, \cdots, i_{M-N+1}}^{M} \prod_{m=i_{1}}^{i_{M-N+1}}\left(\eta^{\prime}+\sum_{i_{1}=1}^{N} \frac{\lambda_{i_{1}, m}^{-N_{r}}}{N_{r} !}\right),
$$

and $\eta^{\prime}=0$ if $N_{d} \neq 1$ and $\eta^{\prime}=\lambda_{m}^{-N_{r}}$ if $N_{d}=1$.

Case 3: $N_{d}=N_{r}=\tilde{N}$. In this case, the dominant terms can be obtained when $v=\tau$ in (32) i.e., $\mathcal{P}_{\text {out }_{2}^{\infty}}^{\mathcal{A}}=$ $\sum_{\tau=1}^{N}\left(\Phi^{\infty}(N-\tau) F_{\gamma_{(\tau)}}^{\infty}\left(\gamma_{t h}\right)\right)$ and is given by

$$
\mathcal{P}_{\text {out }_{2}^{\infty}}^{\mathcal{A}}=\Xi_{2^{\prime \prime}}^{\mathcal{A}}\left(\frac{\gamma_{t h}}{\bar{\gamma}}\right)^{(M+1) \tilde{N}}
$$

where

$$
\begin{aligned}
\Xi_{2^{\prime \prime}}^{\mathcal{A}}=\sum_{\tau=1}^{N}\left(\sum_{i_{1}, i_{2}, \cdots, i_{\tau}}^{N}\left(\prod_{n=i_{1}}^{i_{\tau}} \frac{\lambda_{n}^{-\tilde{N}}}{\tilde{N} !}\right)\right. & \left.\sum_{i_{1}, i_{2}, \cdots, i_{M-\tau+1}}^{M} \prod_{m=i_{1}}^{i_{M-\tau+1}}\left(\sum_{i_{1}=1}^{N} \frac{\lambda_{i_{1}}^{-\tilde{N}}}{\tilde{N} !}\right)\right) .
\end{aligned}
$$

Using (1), the diversity order and the coding gain of MIMONCC system with RS Strategy $\mathcal{A}$ when $N>K$ and $N \leq K$ are respectively given by

$$
G_{d_{1}}^{\mathcal{A}}=\min \left\{M N_{r}, K N_{d}\right\}+N_{d}
$$

TABLE I

DiVERSITY ORDER OF RS STRATEGY $\mathcal{A}$

\begin{tabular}{ccc}
\hline \multicolumn{3}{c}{ Diversity Order for $N>K(39)$} \\
\hline$N_{d}=N_{r}=\tilde{N}$ & $N_{r}>N_{d}$ or $\left(N_{d}>N_{r}, \frac{M}{K}>\frac{N_{d}}{N_{r}}\right)$ & $N_{d}>N_{r}, \frac{M}{K}<\frac{N_{d}}{N_{r}}$ \\
\hline$(K+1) \tilde{N}$ & $(K+1) N_{d}$ & $M N_{r}+N_{d}$ \\
\hline \multicolumn{3}{c}{ Diversity Order for $N \leq K(41)$} \\
\hline$N_{d}=N_{r}=\tilde{N}$ & $N_{r}>N_{d}$ & $N_{r}<N_{d}$ \\
\hline$(M+1) \tilde{N}$ & $(M-N+1) N_{r}+N N_{d}$ & $M N_{r}+N_{d}$ \\
\hline
\end{tabular}

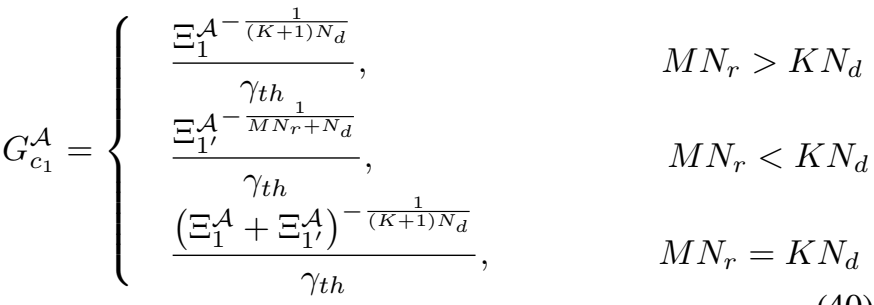$$
G_{d_{2}}^{\mathcal{A}}=(M+1) N_{r}+\min \left\{N_{d}-N_{r}, N\left(N_{d}-N_{r}\right)\right\},
$$

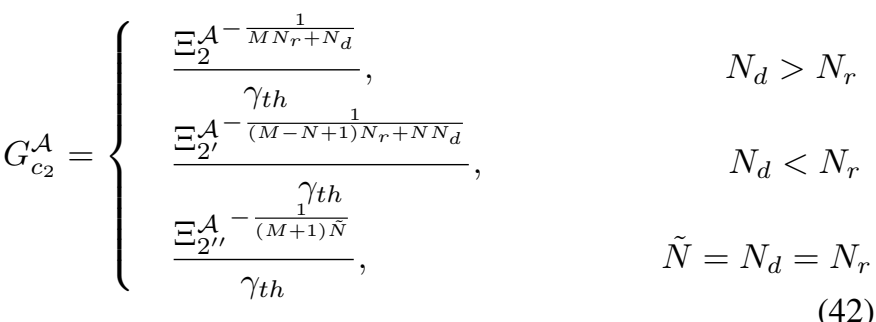

Special Case 1. Single best RS is a special case of $N>K$ when $K=1$. Furthermore, when $\tilde{N}=N_{d}=N_{r}=1$, the diversity orders given by (39) and (41) reduce to $K+1$, and $M+1$ for $N>K$, and $N \leq K$, respectively. Therefore, our diversity order analysis is a generalized version of those earlier presented in [17], [18] and coincide with them when single-antenna terminals are considered.

To have further insights and guidelines for practical implementation obtained through our diversity analyses, the diversity orders i.e., (39) and (41) for different values of system parameters $N_{d}, N_{r}, N, M$, and $K$ are provided in Table I. These insights and guidelines may be useful in designing practical MIMO-NCC systems with RS protocols. In the following we provide some remarks.

- The diversity order is equal to $M n_{r}+N_{d}$ for both $N>$ $K,\left(N_{d}>N_{r}, \frac{M}{K}<\frac{N_{d}}{N_{r}}\right)$ and $N \leq K$ when $N_{r}<N_{d}$.

- The diversity is always independent of selected relays, $K$, when $N \leq K$. Thus, increasing number of selected relays not only does not provide any performance gain but also decreases the system throughput.

- Interestingly, but counter-intuitively, for $N \leq K\left(N_{r}>\right.$ $N_{d}$ ), the diversity order is a function of $N$. This is the only case that increasing the number of sources (while keeping $M, N_{r}$, and $N_{d}$ fixed) leads to the diversity loss.

- It can be seen from Table I that the achievable diversity order for SRS (i.e., when $K=1$ ) depends on the number of relays $M$ when $\frac{N_{d}}{N_{r}}>M$ i.e., $M N_{r}+N_{d}$. However, for single-antenna NCC system the diversity order is always two irrespective of the number of relays [17]-[19].

- If a single-antenna destination is used i.e., $N_{d}=1$, the diversity order for the case of $N>K$ reduces to 
Broadcasting Phase

\begin{tabular}{|c|c|c|}
\hline $\begin{array}{c}S_{1} \\
\text { transmits }\end{array}$ & $\ldots$ & $S_{N}$ \\
\hline$\triangleleft$ & transmits \\
\hline 1
\end{tabular}

$N$ Time Slots

(a)

Broadcasting Phase

Relaying Phase

\begin{tabular}{|c|c|c|c|c|c|}
\hline $\begin{array}{c}S_{1} \\
\text { transmits }\end{array}$ & $\cdots$ & $\begin{array}{c}S_{N} \\
\text { transmits }\end{array}$ & $\begin{array}{c}R_{(1)}^{*} \in \mathcal{D} \\
\text { transmits }\end{array}$ & $\cdots$ & $\begin{array}{c}R_{(K)}^{*} \in \mathcal{D} \\
\text { transmits }\end{array}$ \\
\hline
\end{tabular}

$N$ Time Slots

(b)

$K$ Time Slots

Fig. 1. Time-resource allocation for RS Strategy $\mathcal{B}_{1}$ (a) $0 \leq l<K$, (b) $K \leq l \leq M$.

$K+1$. Therefore, increasing $M$ or $N_{r}$ does not improve the diversity order. However, for $N \leq K$, adding relays and the antennas at relays increases diversity order. This reveals that in order to take benefit from relays and the antennas at relays when $N>K$, the destination should be equipped with multiple antennas such that it satisfies the condition $\frac{N_{d}}{N_{r}}>\frac{M}{K}$.

\section{RS STRATEGy $\mathcal{B}$}

We have just derived the outage probability, diversity order, and coding gain of RS Strategy $\mathcal{A}$. However, as mentioned before, this RS strategy may not be always practical and feasible for a network with large number of branches. It requires large signaling overheads due to the need of global CSI of all source-relay and relay-destination links (8) for RS process. We thus propose a new RS strategy for NCC system based on local CSI of the relay-to-destination channels, and it can be described as follows:

In Strategy $\mathcal{B}$, after the end of the first phase, the relays that have correctly decoded all the packets from $N$ sources form a decoding set $\mathcal{D}$. Mathematically, this set can be written as $\mathcal{D} \triangleq\left\{m: \gamma_{n, m}>\gamma_{t h}, \forall n\right\}$. Let $\mathcal{D}_{l}$ be a decoding set with $l$ relays. In RS Strategy $\mathcal{B}, K$ out of $l$ relays in $\mathcal{D}_{l}$ are selected to transmit their encoded sources' packets to the destination. In particular, the $v$ th best relay $R_{(v)}^{*}(v=1,2, \cdots, K)$ is selected according to the following policy:

$$
(v)=\operatorname{argmax}_{m \in \mathcal{D}_{l}}^{v \text { th }}\left\{\gamma_{m}^{(\hat{i})}\right\}
$$

which implies that $K$ best relays belonging to $\mathcal{D}_{l}$ with the highest instantaneous SNR of the relay-to-destination channels are chosen to cooperate. ${ }^{3}$ The destination will thus receive some network-coded packets as well as original packets directly from the sources. If it receives less than $N$ correct packets, an outage occurs.

The RS policy under Strategy $\mathcal{B}(43)$ depends on $\mathcal{D}_{l}$. Since the number of selected relays, $K$, is a priory fixed number and the size of $\mathcal{D}_{l}, l$, is randomly varying with fluctuation

\footnotetext{
${ }^{3}$ Unlike RS Strategy $\mathcal{A}$, in RS Strategy $\mathcal{B}$, the relays that decode incorrectly are not allowed to take part in cooperation. Hence, no error propagation occurs.
}

of channels (i.e., $0 \leq l \leq M)$, it is possible that $l<K^{4}$ Two strategies can be made when $l<K$. In this section, we investigate the performance of these two strategies namely, Strategy $\mathcal{B}_{1}$ and Strategy $\mathcal{B}_{2}$.

\section{A. RS Strategy $\mathcal{B}_{1}$}

For RS under Strategy $\mathcal{B}_{1}$, if the number of relays in $\mathcal{D}_{l}$ is less than $K$, the $\mathrm{RS}$ process is not performed and all the relays belonging to $\mathcal{D}_{l}$ keep silent until the next round of cooperation begins. On the other hand, if the size of $\mathcal{D}_{l}$ is greater than or equal to $K$, then $K$ best relays out of $l$ relays in $\mathcal{D}_{l}$ are selected to transmit during the relaying phase. Fig. 1 depicts the time-resource allocation for RS Strategy $\mathcal{B}_{1}$.

1) Outage Probability: In this section, we derive closedform expressions of the outage probability for MIMO-NCC systems with $\mathrm{RS}$ under strategy $\mathcal{B}_{1}$. The asymptotic outage expressions are also derived to obtain the achievable diversity order and coding gain.

The overall outage probability of the system under Strategy $\mathcal{B}_{i}(i=1,2)$ can be expressed as

$$
\mathcal{P}_{\text {out }}^{\mathcal{B}_{i}}=\sum_{l=0}^{M} \sum_{\mathcal{D}_{l}} \operatorname{Pr}\left\{\mathcal{O}_{\mathcal{B}_{i}} \mid \mathcal{D}_{l}\right\} \Psi(l)
$$

where $\operatorname{Pr}\left\{\mathcal{O}_{\mathcal{B}_{i}} \mid \mathcal{D}_{l}\right\}$ represents the outage probability conditioned on $\mathcal{D}_{l}$ and $\Psi(l)=\operatorname{Pr}\left\{\mathcal{D}_{l}\right\}$. In the following, we proceed to obtain the outage probability of Strategy $\mathcal{B}_{1}$ by deriving $\Psi(l)$ and $\operatorname{Pr}\left\{\mathcal{O}_{\mathcal{B}_{1}} \mid \mathcal{D}_{l}\right\}$.

In (44), $\Psi(l)$ can be written as

$$
\Psi(l)=\prod_{m^{\prime} \in \mathcal{D}_{l}} \mathrm{P}\left\{\mathcal{S}_{m^{\prime}}\right\} \prod_{m \notin \mathcal{D}_{l}}\left(1-\mathrm{P}\left\{\mathcal{S}_{m}\right\}\right),
$$

which can be rewritten as

$$
\Psi(l)=\sum_{k=0}^{l}(-1)^{k} \sum_{i_{1}, \cdots, i_{k} \in \mathcal{D}_{l}} \prod_{m^{\prime}=i_{1}}^{i_{k}} \mathrm{P}^{\prime}\left\{\mathcal{S}_{m^{\prime}}\right\} \prod_{m \notin \mathcal{D}_{l}} \mathrm{P}^{\prime}\left\{\mathcal{S}_{m}\right\},
$$

\footnotetext{
${ }^{4}$ The decoding set $\mathcal{D}_{l}$, is the set of relays that have been successful in decoding all sources' packets. Hence, the size of $\mathcal{D}_{l}, l$, is not controllable and is determined by channel fading, which is random. Therefore, it is impossible to control $l$ to be always greater than number of selected relays.
} 
where $\mathrm{P}^{\prime}\left\{\mathcal{S}_{m}\right\}=1-\mathrm{P}\left\{\mathcal{S}_{m}\right\}$ and $\mathrm{P}\left\{\mathcal{S}_{m}\right\}$ is the probability that relay $R_{m}$ successfully decodes all $N$ sources' packets. This can be written as

$$
\mathrm{P}\left\{\mathcal{S}_{m}\right\}=1+\sum_{k=1}^{N}(-1)^{k} \sum_{\substack{i_{1}, i_{2}, \cdots, i_{k} \\ i_{1}<i_{2}<\cdots<i_{k}}}^{N} \prod_{n=i_{1}}^{i_{k}} F_{\gamma_{n, m}}\left(\gamma_{t h}\right) .
$$

Let $\gamma_{(v)}=\max _{m \in \mathcal{D}_{l}}^{v \text { th }}\left\{\gamma_{m}^{(\hat{i})}\right\}$ denote the SNR of relay $R_{(v)}^{*}$. Then, we have

$$
\begin{gathered}
\operatorname{Pr}\left\{\gamma_{(v) \mid \mathcal{D}_{l}}<\gamma, \gamma_{(v-1) \mid \mathcal{D}_{l}}>\gamma\right\}= \\
\operatorname{Pr}\left\{\left(v-1 \text { of } \gamma_{m}^{(\hat{i})}, \mathrm{s}>\gamma\right) \bigcap\left(l-v+1 \text { of } \gamma_{m}^{(\hat{i})}, \mathrm{s}<\gamma\right)\right\} .
\end{gathered}
$$

Since SNRs of the relays are independent random variables, (48) can be derived as

$$
F_{\gamma_{(v) \mid l}}(\gamma)=\sum_{k=1}^{v} \mathcal{C}_{l-v+1}^{l-v+k}(-1)^{k-1} \sum_{\substack{i_{1}, i_{2}, \cdots, i_{l-v+k} \in \mathcal{D}_{l} \\ i_{l-v+k}}} F_{\gamma_{m}^{(\hat{)})}}(\gamma) .
$$

Under RS Strategy $\mathcal{B}_{1}$, the destination potentially receives (i) $N$ packets if $0 \leq l<K$ and (ii) $N+K$ packets if $K \leq l \leq$ $M$. In both cases an outage occurs if less than $N$ packets are decoded correctly by the destination. Let $K_{\mathrm{op}}^{\mathcal{B}} \leq K$ denote the number of selected relays whose relay-to-destination channels are not in outage. Now, depending on $l$, we have the following events which lead to th outage of the system:

(i) $0 \leq l<K$ : When the number of available relays in $\mathcal{D}_{l}$ is less than $K$, all $l$ relays in $\mathcal{D}_{l}$ remain silent. Therefore, the destination receives only $N$ packets from direct transmissions through the source-to-destination channels. An outage occurs if at most $N-1$ links from $N$ sourceto-destination links be operational i.e., $N_{\text {op }}<N$. We denote this event by $\mathcal{O}_{\mathcal{B}_{1}}^{(1)}$.

(ii) $K \leq l \leq M$ : If the number of available relays in $\mathcal{D}_{l}$ is equal or more than $K$, then $\mathrm{RS}$ is performed. In this case, we have the following two outage events:

- $\mathcal{O}_{\mathcal{B}_{1}}^{(2)} \mid \mathcal{D}_{l}$ represents the outage events when $N_{\mathrm{op}}+$ $K<N$.

- $\mathcal{O}_{\mathcal{B}_{1}}^{\left(2^{\prime}\right)} \mid \mathcal{D}_{l}$ corresponds to the outage events when $N_{\text {op }}+K \geq N$ but $N_{\text {op }}+K_{\text {op }}^{\mathcal{B}}<N$.
Thus, the overall outage event in (44) can be calculated as

$$
\mathcal{O}_{\mathcal{B}_{1}}\left|\mathcal{D}_{l}=\mathcal{O}_{\mathcal{B}_{1}}^{(1)} \bigcup \mathcal{O}_{\mathcal{B}_{1}}^{(2)}\right| \mathcal{D}_{l} \bigcup \mathcal{O}_{\mathcal{B}_{1}}^{\left(2^{\prime}\right)} \mid \mathcal{D}_{l}
$$

Using (50), the outage probability of the system conditioned on $\mathcal{D}_{l}$ is given by

$$
\operatorname{Pr}\left\{\mathcal{O}_{\mathcal{B}_{1}} \mid \mathcal{D}_{l}\right\}=\operatorname{Pr}\left\{\mathcal{O}_{\mathcal{B}_{1}}^{(1)}\right\}+\operatorname{Pr}\left\{\mathcal{O}_{\mathcal{B}_{1}}^{(2)} \mid \mathcal{D}_{l}\right\}+\operatorname{Pr}\left\{\mathcal{O}_{\mathcal{B}_{1}}^{\left(2^{\prime}\right)} \mid \mathcal{D}_{l}\right\}
$$

The term $\operatorname{Pr}\left\{\mathcal{O}_{\mathcal{B}_{1}}^{(1)}\right\}$ can be obtained as

$$
\operatorname{Pr}\left\{\mathcal{O}_{\mathcal{B}_{1}}^{(1)}\right\}=\sum_{\tau=0}^{N-1} \Phi(\tau), \quad 0 \leq l<K,
$$

where $\Phi(\tau)$ is given by (17).

Furthermore, $\operatorname{Pr}\left\{\mathcal{O}_{\mathcal{B}_{1}}^{(2)} \mid \mathcal{D}_{l}\right\}+\operatorname{Pr}\left\{\mathcal{O}_{\mathcal{B}_{1}}^{\left(2^{\prime}\right)} \mid \mathcal{D}_{l}\right\}$ when $N>K$ can be formulated as

$$
\begin{aligned}
& \operatorname{Pr}\left\{\mathcal{O}_{\mathcal{B}_{1}}^{(2)} \mid \mathcal{D}_{l}\right\}+\operatorname{Pr}\left\{\mathcal{O}_{\mathcal{B}_{1}}^{\left(2^{\prime}\right)} \mid \mathcal{D}_{l}\right\}=\sum_{\tau=0}^{N-K-1} \Phi(\tau) \\
& +\sum_{\tau=1}^{K}\left(\Phi(N-\tau) \sum_{v=1}^{\tau} F_{\gamma_{(v) \mid l}}\left(\gamma_{t h}\right)\right), \quad K \leq l \leq M,
\end{aligned}
$$

where $F_{\gamma_{(v) \mid l}}(\gamma)$ is given by (49).

Finally, substituting (52), (53), and (46) into (44), one can obtain the exact outage probability of RS Strategy $\mathcal{B}_{1}$ when $N>K$. This can be expressed in the closed-form expression given by (54). Similarly, the outage probability of the system when $N \leq K$ is derived as (55).

2) Asymptotic Analysis: $\mathrm{P}^{\prime}\left\{\mathcal{S}_{m}\right\}$ for high SNRs is approximated as $\mathrm{P}^{\prime \infty}\left\{\mathcal{S}_{m}\right\}=\sum_{i_{1}=1}^{N}\left(\frac{\beta_{i_{1}, m}^{N_{r}}}{N_{r} !}\right)$. Substituting this expression into (46) and discarding higher order terms, $\Psi(l)$ can be approximated as

$$
\Psi^{\infty}(l)=\prod_{\substack{m=i_{1} \\ m \notin \mathcal{D}_{l}}}^{i_{M-l}}\left(\sum_{i_{1}=1}^{N} \frac{\beta_{i_{1}, m}^{N_{r}}}{N_{r} !}\right) .
$$

Furthermore, we obtain (49) in high SNRs as

$$
F_{\gamma_{(v) \mid l}}^{\infty}\left(\gamma_{t h}\right)=\sum_{i_{1}, i_{2}, \cdots, i_{l-v+1} \in \mathcal{D}_{l}} \prod_{m=i_{1}}^{i_{l-v+1}}\left(\frac{\beta_{m}^{N_{d}}}{N_{d} !}\right)^{N_{r}} .
$$

Plugging (56), (57), and (23) into (54) and then retaining the dominant terms, we have

$$
\begin{aligned}
\mathcal{P}_{\text {out }_{1}^{\infty}}^{\mathcal{B}_{1}} & =\sum_{\mathcal{D}_{K-1}} \Phi^{\infty}(N-1) \Psi^{\infty}(K-1) \\
& +\Phi^{\infty}(N-K-1) \Psi^{\infty}(M) .
\end{aligned}
$$

$$
\begin{aligned}
& \mathcal{P}_{\text {out }_{1}}^{\mathcal{B}_{1}}=\underbrace{\sum_{l=0}^{K-1} \sum_{\mathcal{D}_{l}}\left(\sum_{\tau=0}^{N-1} \Phi(\tau)\right) \Psi(l)}_{\operatorname{Pr}\left\{\mathcal{O}_{\mathcal{B}_{1}}^{(1)}\right\}}+\underbrace{\sum_{l=K}^{M} \sum_{\mathcal{D}_{l}}\left(\sum_{\tau=0}^{N-K-1} \Phi(\tau)\right) \Psi(l)}_{\operatorname{Pr}\left\{\mathcal{O}_{\mathcal{B}_{1}}^{(2)}\right\}}+\underbrace{\sum_{l=K}^{M} \sum_{\mathcal{D}_{l}}\left(\sum_{\tau=1}^{K} \Phi(N-\tau) \sum_{v=1}^{\tau} F_{\gamma_{(v) \mid l}}\left(\gamma_{t h}\right)\right) \Psi(l)}_{\operatorname{Pr}\left\{\mathcal{O}_{\mathcal{B}_{1}^{\left(2^{\prime}\right)}}\right\}} . \\
& \mathcal{P}_{\text {out }_{2}}^{\mathcal{B}_{1}}=\underbrace{\sum_{l=0}^{K-1} \sum_{\mathcal{D}_{l}}\left(\sum_{\tau=0}^{N-1} \Phi(\tau)\right) \Psi(l)}_{\operatorname{Pr}\left\{\mathcal{O}_{\mathcal{B}_{1}}^{(1)}\right\}}+\underbrace{\sum_{l=K}^{M} \sum_{\mathcal{D}_{l}}\left(\sum_{\tau=1}^{N} \Phi(N-\tau) \sum_{v=1}^{\tau} F_{\gamma_{(v) \mid l}}\left(\gamma_{t h}\right)\right) \Psi(l)}_{\operatorname{Pr}\left\{\mathcal{O}_{\mathcal{B}_{1}}^{\left(2^{\prime}\right)}\right\}} .
\end{aligned}
$$


Based on the relationship between $(M-K+1) N_{r}$ and $K N_{d}$, (54) in high SNRs can be derived as follows:

Case 1: $(M-K+1) N_{r}<K N_{d}$. In this case, $\mathcal{P}_{\text {out }_{1}^{\infty}}^{\mathcal{B}_{1}}$ is determined by the first term in (58) as $\mathcal{P}_{\text {out }_{1}^{\infty}}^{\mathcal{B}_{1}}=\sum_{\mathcal{D}_{K-1}} \Phi^{\infty}(N-$ 1) $\Psi^{\infty}(K-1)$. This is given by

$$
\mathcal{P}_{\text {out }_{1}^{\infty}}^{\mathcal{B}_{1}}=\Xi_{1}^{\mathcal{B}_{1}}\left(\frac{\gamma_{t h}}{\bar{\gamma}}\right)^{(M-K+1) N_{r}+N_{d}},
$$

where

$$
\Xi_{1}^{\mathcal{B}_{1}}=\sum_{\mathcal{D}_{K-1}}\left(\sum_{i_{1}=1}^{N} \frac{\lambda_{i_{1}}^{-N_{d}}}{N_{d} !} \prod_{m=i_{1}}^{i_{M-K+1}}\left(\sum_{i_{1}=1}^{N} \frac{\lambda_{i_{1}, m}^{-N_{r}}}{N_{r} !}\right)\right) .
$$

Case 2: $(M-K+1) N_{r}>K N_{d}$. In this case, $\mathcal{P}_{\text {out }_{1}^{\infty}}^{\mathcal{B}_{1}}$ is determined by the second term in (58) i.e., $\mathcal{P}_{\text {out }_{1}^{\infty}}^{\mathcal{B}_{1}}=\Phi^{\infty}(N-$ $K-1) \Psi^{\infty}(M)$. This can be written as

$$
\mathcal{P}_{\text {out }_{1}^{\infty}}^{\mathcal{B}_{1}}=\Xi_{1^{\prime}}^{\mathcal{B}_{1}}\left(\frac{\gamma_{t h}}{\bar{\gamma}}\right)^{(K+1) N_{d}},
$$

where

$$
\Xi_{1^{\prime}}^{\mathcal{B}_{1}}=\sum_{i_{1}, i_{2}, \cdots, i_{K+1}}^{N}\left(\prod_{n=i_{1}}^{i_{K+1}} \frac{\lambda_{n}^{-N_{d}}}{N_{d} !}\right) .
$$

Case 3: $(M-K+1) N_{r}=K N_{d}$. In this case, $\mathcal{P}_{\text {out }_{1}^{\infty}}^{\mathcal{B}_{1}}$ is determined by both the first and second terms in (58) as $\mathcal{P}_{\text {out }_{1}^{\infty}}^{\mathcal{B}_{1}}=\sum_{\mathcal{D}_{K-1}} \Phi^{\infty}(N-1) \Psi^{\infty}(K-1)+\Phi^{\infty}(N-K-$ 1) $\Psi^{\infty}(M)$.

Now, we proceed to obtain the asymptotic outage expression when $N \leq K$. Substituting (56), (57), and (23) into (55), and then keeping the dominant terms, it can be checked that the asymptotic outage is independent of the relationship between system parameters and is always equal to $\mathcal{P}_{\text {out }_{2}^{\infty}}^{\mathcal{B}_{1}}=$ $\sum_{\mathcal{D}_{K-1}} \Phi^{\infty}(N-1) \Psi^{\infty}(K-1)$. This can be expressed as

$$
\mathcal{P}_{\text {out }_{2}^{\infty}}^{\mathcal{B}_{1}}=\Xi_{2}^{\mathcal{B}_{1}}\left(\frac{\gamma_{t h}}{\bar{\gamma}}\right)^{(M-K+1) N_{r}+N_{d}},
$$

where

$$
\Xi_{2}^{\mathcal{B}_{1}}=\sum_{\mathcal{D}_{K-1}}\left(\sum_{i_{1}=1}^{N} \frac{\lambda_{i_{1}}^{-N_{d}}}{N_{d} !} \prod_{m=i_{1}}^{i_{M-K+1}}\left(\sum_{i_{1}=1}^{N} \frac{\lambda_{i_{1}, m}^{-N_{r}}}{N_{r} !}\right)\right) .
$$

Invoking (1), the diversity order and the coding gain of MIMONCC system with RS Strategy $\mathcal{B}_{1}$ for $N>K$ and $N \leq K$ are respectively given by

$$
\begin{aligned}
& G_{d_{1}}^{\mathcal{B}_{1}}=\min \left\{(M-K+1) N_{r}, K N_{d}\right\}+N_{d}, \\
& G_{c_{1}}^{\mathcal{B}_{1}}= \begin{cases}\frac{\Xi_{1}^{\mathcal{B}_{1}-\frac{1}{(M-K+1) N_{r}+N_{d}}},}{\gamma_{t h}}, & (M-K+1) N_{r}<K N_{d} \\
\frac{\Xi_{1^{\prime}}^{\mathcal{B}_{1}}-\frac{1}{(K+1) N_{d}}}{\gamma_{t h}}, & (M-K+1) N_{r}>K N_{d} \\
\frac{\left(\Xi_{1}^{\mathcal{B}_{1}}+\Xi_{1^{\prime}}^{\mathcal{B}_{1}}\right)^{-\frac{1}{(K+1) N_{d}}}}{\gamma_{t h}}, & (M-K+1) N_{r}=K N_{d}\end{cases}
\end{aligned}
$$

TABLE II

Diversity ORDER OF RS STRATEgy $\mathcal{B}_{1}$

\begin{tabular}{ccc}
\hline \multicolumn{3}{c}{ Diversity Order for $N>K(65)$} \\
\hline$K<\frac{(M+1) N_{r}}{N_{r}+N_{d}}$ & $K=\frac{(M+1) N_{r}}{N_{r}+N_{d}}$ & $K>\frac{(M+1) N_{r}}{N_{r}+N_{d}}$ \\
\hline$(K+1) N_{d}$ & $(K+1) N_{d}$ & $(M-K+1) N_{r}+N_{d}$ \\
\hline & Diversity Order for $N \leq K(67)$ \\
\hline & $(M-K+1) N_{r}+N_{d}$ \\
\hline
\end{tabular}

$$
\begin{aligned}
G_{d_{2}}^{\mathcal{B}_{1}} & =(M-K+1) N_{r}+N_{d}, \\
G_{c_{2}}^{\mathcal{B}_{1}} & =\frac{\Xi_{2}^{\mathcal{B}_{1}}-\frac{1}{(M-K+1) N_{r}+N_{d}}}{\gamma_{t h}} .
\end{aligned}
$$

Comparing (65) and (67) with (39) and (41), one can realize that the achievable diversity order of RS Strategy $\mathcal{B}_{1}$ is always equal or less than that of Strategy $\mathcal{A}$.

The diversity orders of Strategy $\mathcal{B}_{1}$ i.e., (65) and (67) for different values of $N_{d}, N_{r}, M$, and $K$ are provided in Table II. Based on Table II, the following insights are highlighted:

- The diversity order is a function of all system parameters except for the number of sources $N$. Accordingly, increasing or decreasing the number of sources does not change the diversity.

- For the case of $K<\frac{(M+1) N_{r}}{N_{r}+N_{d}}$, the diversity order and the coding gain are exactly similar to that of Strategy $\mathcal{A}$ when $N>K$ and $M N_{r}>K N_{d}$.

- Interestingly, but counter-intuitively, an increase in the number of selected relays has negative impact on the diversity when $K>\frac{(M+1) N_{r}}{N_{r}+N_{d}},(N>K)$ or $N \leq K$. This contradicts our expectation that selecting more relays improves the system performance.

- The diversity order is independent of $M$ and $N_{r}$ when $K \leq \frac{(M+1) N_{r}}{N_{r}+N_{d}}$. Thus, adding relays or the antennas at relays does not improve the performance.

\section{B. RS Strategy $\mathcal{B}_{2}$}

The RS Strategy $\mathcal{B}_{1}$ is not capable of achieving the diversity orders similar to Strategy $\mathcal{A}$. The assumption that the relays in $\mathcal{D}_{l}$ have to be silent when $0 \leq l<K$ may be too restrictive for such scheme. In this section, we relax this assumption and assume that the relays in $\mathcal{D}_{l}$ will cooperate without RS when $0 \leq l \leq K$, while RS is carried out when $k<l \leq M$. Fig. 2 depicts time-resource allocation for RS Strategy $\mathcal{B}_{2}$.

1) Outage Probability: Recall that the outage probability of Strategy $\mathcal{B}_{2}$ is given by (44), where $\Psi(l)$ has been derived in the previous section (46). In the following, we proceed to obtain $\operatorname{Pr}\left\{\mathcal{O}_{\mathcal{B}_{2}} \mid \mathcal{D}_{l}\right\}$.

In RS Strategy $\mathcal{B}_{2}$, the destination potentially receives (i) $N+l$ packets if $0 \leq l \leq K$ and (ii) $N+K$ packets if $K<l \leq M$. An outage occurs if fewer than $N$ packets are decoded by the destination. Let $l_{\mathrm{op}} \leq l$ denote the number of relays in $\mathcal{D}_{l}$ whose relay-to-destination channels are not in outage. Depending on $l$, we have the following outage events:

(i) $0 \leq l \leq K$ : When the number of available relays in $\mathcal{D}_{l}$ is less than or equal to $K$, all $l$ relays transmit without 


\begin{tabular}{|c|c|c|c|c|}
\hline $\begin{array}{c}S_{1} \\
\text { transmits }\end{array}$ & $\ldots$ & $\begin{array}{c}S_{N} \\
\text { transmits }\end{array}$ & $\begin{array}{l}R_{1} \in \mathcal{D} \\
\text { transmits }\end{array}$ & $\begin{array}{l}R_{l} \in \mathcal{D} \\
\text { transmits }\end{array}$ \\
\hline \multicolumn{3}{|c|}{$N$ Time Slots } & \multicolumn{2}{|c|}{$l$ Time Slots } \\
\hline \multicolumn{3}{|c|}{ Broadcasting Phase } & \multicolumn{2}{|c|}{ Relaying Phase } \\
\hline $\begin{array}{c}S_{1} \\
\text { transmits }\end{array}$ & $\ldots$ & $\begin{array}{c}S_{N} \\
\text { transmits }\end{array}$ & $\begin{array}{c}R_{(1)}^{*} \in \mathcal{D} \\
\text { transmits }\end{array}$ & $\begin{array}{c}R_{(K)}^{*} \in \mathcal{D} \\
\text { transmits }\end{array}$ \\
\hline
\end{tabular}

(b)

Fig. 2. Time-resource allocation for RS Strategy $\mathcal{B}_{2}$ (a) $0 \leq l \leq K$, (b) $K<l \leq M$.

selection. In this case, an outage occurs if at most $N-1$ links from $N$ source-to-destination links and $l$ relay-todestination links be operational i.e., $N_{\mathrm{op}}+l_{\mathrm{op}}<N$. This event is denoted as $\mathcal{O}_{\mathcal{B}_{2}}^{(1)} \mid \mathcal{D}_{l}$.

(ii) $K<l \leq M$ : If the number of available relays in $\mathcal{D}_{l}$ is more than $K$, then RS is performed. Therefore, two outage events happen:

- $\mathcal{O}_{\mathcal{B}_{2}}^{(2)} \mid \mathcal{D}_{l}$ represents the outage events when $N_{\text {op }}<$ $N-K$.

- $\mathcal{O}_{\mathcal{B}_{2}}^{\left(2^{\prime}\right)} \mid \mathcal{D}_{l}$ corresponds to the outage events when $N_{\text {op }} \geq N-K$ but $N_{\text {op }}+K_{\text {op }}^{\mathcal{B}}<N$.

The overall outage probability conditioned on $\mathcal{D}_{l}$ in (44) can be then written as

$\operatorname{Pr}\left\{\mathcal{O}_{\mathcal{B}_{2}} \mid \mathcal{D}_{l}\right\}=\operatorname{Pr}\left\{\mathcal{O}_{\mathcal{B}_{2}}^{(1)} \mid \mathcal{D}_{l}\right\}+\operatorname{Pr}\left\{\mathcal{O}_{\mathcal{B}_{2}}^{(2)} \mid \mathcal{D}_{l}\right\}+\operatorname{Pr}\left\{\mathcal{O}_{\mathcal{B}_{2}}^{\left(2^{\prime}\right)} \mid \mathcal{D}_{l}\right\}$

In (69), $\operatorname{Pr}\left\{\mathcal{O}_{\mathcal{B}_{2}}^{(1)} \mid \mathcal{D}_{l}\right\}$ can be formulated as

$$
\operatorname{Pr}\left\{\mathcal{O}_{\mathcal{B}_{2}}^{(1)} \mid \mathcal{D}_{l}\right\}=\sum_{m=0}^{l} \Theta(m \mid l) \sum_{\tau=0}^{N-1-m} \Phi(\tau), 0 \leq l \leq K,
$$

where $\Theta(m \mid l)$ is the probability that $m$ relays out of $l$ relays be operational and can be written as

$$
\Theta(m \mid l)=\sum_{k=1}^{m+1} \mathcal{C}_{l-m}^{l+k-m-1}(-1)^{k-1} \sum_{\substack{i_{1}, i_{2}, \cdots, i_{l+k-m-1} \in \mathcal{D}_{l} \\ i_{l+k-m-1}}} F_{\gamma_{m}^{(\hat{i})}}\left(\gamma_{t h}\right) .
$$

On the other hand, $\operatorname{Pr}\left\{\mathcal{O}_{\mathcal{B}_{2}}^{(2)} \mid \mathcal{D}_{l}\right\}+\operatorname{Pr}\left\{\mathcal{O}_{\mathcal{B}_{2}}^{\left(2^{\prime}\right)} \mid \mathcal{D}_{l}\right\}$ for $N>K$ can be derived as

$$
\begin{aligned}
& \operatorname{Pr}\left\{\mathcal{O}_{\mathcal{B}_{2}}^{(2)} \mid \mathcal{D}_{l}\right\}+\operatorname{Pr}\left\{\mathcal{O}_{\mathcal{B}_{2}}^{\left(2^{\prime}\right)} \mid \mathcal{D}_{l}\right\}=\sum_{\tau=0}^{N-K-1} \Phi(\tau) \\
& +\sum_{\tau=1}^{K}\left(\Phi(N-\tau) \sum_{v=1}^{\tau} F_{\gamma_{(v) \mid l}}\left(\gamma_{t h}\right)\right), \quad K<l \leq M .
\end{aligned}
$$

By plugging (70), (72), and (46) into (44), the exact outage probability for $N>K$ under Strategy $\mathcal{B}_{2}$ can be derived as (73). With similar arguments used above, we obtain the outage probability of the system when $N \leq K$ as (74).
2) Asymptotic Analysis: $\Theta(m \mid l)$ in high SNRs can be approximated as

$$
\Theta^{\infty}(m \mid l)=\sum_{i_{1}, i_{2}, \cdots, i_{l-m} \in \mathcal{D}_{l}} \prod_{m=i_{1}}^{i_{l-m}}\left(\frac{\beta_{m}^{N_{d}}}{N_{d} !}\right)^{N_{r}} .
$$

Substituting (23), (56), (57) and (75) in (73) and ignoring higher order terms, we have

$$
\mathcal{P}_{\text {out }_{1}^{\infty}}^{\mathcal{B}_{2}}=\Psi^{\infty}(0) \Phi^{\infty}(N-1)+\Phi^{\infty}(N-K-1) \Psi^{\infty}(M) .
$$

The asymptotic outage depends on the system parameters. In particular, (73) in high SNRs is derived in the following three cases:

Case 1: $M N_{r}>K N_{d}$. In this case, $\mathcal{P}_{\text {out }_{1}^{\infty}}^{\mathcal{B}_{2}}$ is determined by the second term in (76) as $\mathcal{P}_{\text {out }_{1}^{\infty}}^{\mathcal{B}_{2}}=\Phi^{\infty}(N-K-1) \Psi^{\infty}(M)$. This is expressed as

$$
\mathcal{P}_{\text {out }_{1}^{\infty}}^{\mathcal{B}_{2}}=\Xi_{1}^{\mathcal{B}_{2}}\left(\frac{\gamma_{t h}}{\bar{\gamma}}\right)^{(K+1) N_{d}},
$$

where

$$
\Xi_{1}^{\mathcal{B}_{2}}=\sum_{i_{1}, i_{2}, \cdots, i_{K+1}}^{N}\left(\prod_{n=i_{1}}^{i_{K+1}} \frac{\lambda_{n}^{-N_{d}}}{N_{d} !}\right) .
$$

Case 2: $M N_{r}<K N_{d}$. In this case, $\mathcal{P}_{\text {out }_{1}^{\infty}}^{\mathcal{B}_{2}}$ is determined by the first term in (76) as $\mathcal{P}_{\text {out }_{1}^{\infty}}^{\mathcal{B}_{2}}=\Psi^{\infty}(0) \Phi^{\infty}(N-1)$. This can be written as

$$
\mathcal{P}_{\text {out }_{1}^{\infty}}^{\mathcal{B}_{2}}=\Xi_{1^{\prime}}^{\mathcal{B}_{2}}\left(\frac{\gamma_{t h}}{\bar{\gamma}}\right)^{M N_{r}+N_{d}},
$$

where

$$
\Xi_{1^{\prime}}^{\mathcal{B}_{2}}=\sum_{i_{1}=1}^{N} \frac{\lambda_{i_{1}}^{-N_{d}}}{N_{d} !} \prod_{m=i_{1}}^{i_{M}}\left(\sum_{i_{1}=1}^{N} \frac{\lambda_{i_{1}, m}^{-N_{r}}}{N_{r} !}\right) .
$$

Case 3: $M N_{r}=K N_{d}$. In this case, $\mathcal{P}_{\text {out }_{1}^{\infty}}^{\mathcal{B}_{2}}$ is determined by the first and the second terms in (76), where $\Phi^{\infty}(N-$ $K-1) \Psi^{\infty}(M)$ and $\Psi^{\infty}(0) \Phi^{\infty}(N-1)$ are derived in (77) and (79), respectively.

The outage probability for $N \leq K$ (74) in high SNRs is derived in the following four cases according to the relationship between $N_{d}$ and $N_{r}$. 


$$
\begin{aligned}
& \mathcal{P}_{\text {out }_{1}}^{\mathcal{B}_{2}}=\underbrace{\sum_{l=0}^{K} \sum_{\mathcal{D}_{l}}\left(\sum_{m=0}^{l} \Theta(m \mid l) \sum_{\tau=0}^{N-1-m} \Phi(\tau)\right) \Psi(l)}_{\operatorname{Pr}\left\{\mathcal{O}_{\mathcal{B}_{2}}^{(1)}\right\}}+\underbrace{\sum_{l=K+1}^{M} \sum_{\mathcal{D}_{l}}\left(\sum_{\tau=0}^{N-K-1} \Phi(\tau)\right) \Psi(l)}_{\operatorname{Pr}\left\{\mathcal{O}_{\mathcal{B}_{2}}^{(2)}\right\}} \\
& +\underbrace{\sum_{l=K+1}^{M} \sum_{\mathcal{D}_{l}}\left(\sum_{\tau=1}^{K} \Phi(N-\tau) \sum_{v=1}^{\tau} F_{\gamma_{(v) \mid l}}\left(\gamma_{t h}\right)\right) \Psi(l)}_{\operatorname{Pr}\left\{\mathcal{O}_{\mathcal{B}_{2}}^{\left(2^{\prime}\right)}\right\}} \\
& \mathcal{P}_{\text {out }_{2}}^{\mathcal{B}_{2}}=\underbrace{\sum_{l=0}^{N-1} \sum_{\mathcal{D}_{l}}\left(\sum_{m=0}^{l} \Theta(m \mid l) \sum_{\tau=0}^{N-1-m} \Phi(\tau)\right) \Psi(l)}_{\operatorname{Pr}\left\{\mathcal{O}_{\mathcal{B}_{2}}^{(1)}\right\}}+\underbrace{\sum_{l=N}^{K} \sum_{\mathcal{D}_{l}}\left(\sum_{m=0}^{N-1} \Theta(m \mid l) \sum_{\tau=0}^{N-1-m} \Phi(\tau)\right) \Psi(l)}_{\operatorname{Pr}\left\{\mathcal{O}_{\mathcal{B}_{2}}^{(1)}\right\}} \\
& +\underbrace{\sum_{l=K+1}^{M} \sum_{\mathcal{D}_{l}}\left(\sum_{\tau=1}^{N} \Phi(N-\tau) \sum_{\tau=1}^{\tau} F_{\gamma_{(v) \mid l}}\left(\gamma_{t h}\right)\right) \Psi(l)}_{\operatorname{Pr}\left\{\mathcal{O}_{\mathcal{B}_{2}}^{\left(2^{\prime}\right)}\right\}}
\end{aligned}
$$

Case 1: $N_{d}>N_{r}$. In this case, we have

$$
\mathcal{P}_{\text {out }_{2}^{\infty}}^{\mathcal{B}_{2}}=\Xi_{2}^{\mathcal{B}_{2}}\left(\frac{\gamma_{t h}}{\bar{\gamma}}\right)^{M N_{r}+N_{d}}
$$

where

$$
\Xi_{2}^{\mathcal{B}_{2}}=\sum_{i_{1}=1}^{N} \frac{\lambda_{i_{1}}^{-N_{d}}}{N_{d} !} \prod_{m=i_{1}}^{i_{M}}\left(\sum_{i_{1}=1}^{N} \frac{\lambda_{i_{1}, m}^{-N_{r}}}{N_{r} !}\right) .
$$

Case 2: $N_{d}<N_{r}, N_{d}=1$. In this case, we have

$$
\mathcal{P}_{\text {out }_{2}^{\infty}}^{\mathcal{B}_{2}}=\Xi_{2^{\prime}}^{\mathcal{B}_{2}}\left(\frac{\gamma_{t h}}{\bar{\gamma}}\right)^{(M-N+1) N_{r}+N},
$$

where $\Xi_{2^{\prime}}^{\mathcal{B}_{2}}$ is given by (84).

Case 3: $N_{d}<N_{r}, N_{d} \neq 1$. In this case,

$$
\mathcal{P}_{\text {out }_{2}^{\infty}}^{\mathcal{B}_{2}}=\Xi_{2^{\prime \prime}}^{\mathcal{B}_{2}}\left(\frac{\gamma_{t h}}{\bar{\gamma}}\right)^{(M-N+1) N_{r}+N N_{d}}
$$

where

$$
\Xi_{2^{\prime \prime}}^{\mathcal{B}_{2}}=\sum_{\mathcal{D}_{N-1}}\left(\prod_{n=i_{1}}^{i_{N}} \frac{\lambda_{n}^{-N_{d}}}{N_{d} !} \prod_{m=i_{1}}^{i_{M-N+1}}\left(\sum_{i_{1}=1}^{N} \frac{\lambda_{i_{1}, m}^{-N_{r}}}{N_{r} !}\right)\right) .
$$

Case 4: $N_{d}=N_{r}=\tilde{N}$. For this case, $\mathcal{P}_{\text {out }_{2}^{\infty}}^{\mathcal{B}_{2}}$ can be obtained as

$$
\mathcal{P}_{\text {out }_{2}^{\infty}}^{\mathcal{B}_{2}}=\Xi_{2^{\prime \prime \prime}}^{\mathcal{B}_{2}}\left(\frac{\gamma_{t h}}{\bar{\gamma}}\right)^{(M+1) \tilde{N}}
$$

where

$$
\begin{aligned}
\Xi_{2^{\prime \prime \prime}}^{\mathcal{B}_{2}} & =\sum_{l=0}^{N-1} \sum_{\mathcal{D}_{l}}\left(\sum_{i_{1}, i_{2}, \cdots, i_{l+1}}^{N} \prod_{n=i_{1}}^{i_{l+1}} \frac{\lambda_{n}^{-\tilde{N}}}{\tilde{N} !}\right. \\
& \left.\prod_{m=i_{1}}^{i_{M-l}}\left(\sum_{i_{1}=1}^{N} \frac{\lambda_{i_{1}, m}^{-\tilde{N}}}{\tilde{N} !}\right)\right) .
\end{aligned}
$$

The diversity order and the coding gain of MIMO-NCC system with RS under strategy $\mathcal{B}_{2}$ for $N>K$ and $N \leq K$ are given, respectively, by

$$
G_{d_{1}}^{\mathcal{B}_{2}}=\min \left\{M N_{r}, K N_{d}\right\}+N_{d} .
$$

$$
G_{c_{1}}^{\mathcal{B}_{2}}= \begin{cases}\frac{\Xi_{1}^{\mathcal{B}_{2}}-\frac{1}{(K+1) N_{d}}}{\gamma_{t h}}, & M N_{r}>K N_{d} \\ \frac{\Xi_{1^{\prime}}^{\mathcal{B}_{2}-\frac{1}{M N_{r}+N_{d}}}}{\gamma_{t h}}, & M N_{r}<K N_{d} \\ \frac{\left(\Xi_{1}^{\mathcal{B}_{2}}+\Xi_{1^{\prime}} \mathcal{B}_{2}\right.}{\gamma_{t h}}, & M N_{r}=K N_{d}\end{cases}
$$

$$
G_{d_{2}}^{\mathcal{B}_{2}}=(M+1) N_{r}+\min \left\{N_{d}-N_{r}, N\left(N_{d}-N_{r}\right)\right\} .
$$

$$
G_{c_{2}}^{\mathcal{B}_{2}}=\left\{\begin{array}{lr}
\frac{\Xi_{2}^{\mathcal{B}_{2}}-\frac{1}{M N_{r}+N_{d}}}{\gamma_{t h}}, & N_{d}>N_{r} \\
\frac{\Xi_{2^{\prime}}^{\mathcal{B}_{2}}-\frac{1}{(M-N+1) N_{r}+N}}{\gamma_{t h}}, & N_{d}<N_{r}, N_{d}=1 \\
\frac{\Xi_{2^{\prime \prime}}^{\mathcal{B}_{2}}-\frac{1}{(M-N+1) N_{r}+N N_{d}}}{\boldsymbol{B}_{t h}}, & N_{d}<N_{r}, N_{d} \neq 1 \\
\frac{\Xi_{2^{\prime \prime \prime}}^{\mathcal{B}_{2}}-\frac{\gamma_{t h}}{(M+1) N}}{\gamma_{t h}}, & \tilde{N}=N_{d}=N_{r}
\end{array}\right.
$$

As can be seen form (89) and (91), RS Strategy $\mathcal{B}_{2}$ is capable of achieving diversity orders similar to Strategy $\mathcal{A}$ given by (39) and (41). Furthermore, although the derived outage expressions in (73) and (74) are completely different from (20) and (21), there are exactly the same for all SNR values. Therefore, the proposed RS strategy has the outage performance similar to Strategy $\mathcal{A}$, while it significantly reduces signaling overhead. 


$$
\begin{gathered}
\Xi_{2^{\prime}}^{\mathcal{B}_{2}}=\sum_{\mathcal{D}_{N-1}}\left(\prod_{n=i_{1}}^{i_{N}} \lambda_{n}^{-1} \prod_{m=i_{1}}^{i_{M-N+1}}\left(\sum_{i_{1}=1}^{N} \frac{\lambda_{i_{1}, m}^{-N_{r}}}{N_{r} !}\right)\right)+\sum_{l=N}^{K} \sum_{\mathcal{D}_{l}}\left(\sum_{i_{1}, i_{2}, \cdots, i_{l-N+1} \in \mathcal{D}_{l}} \prod_{m=i_{1}}^{i_{l-N+1}} \lambda_{m}^{-N_{r}} \prod_{n=i_{1}}^{i_{N}} \lambda_{n}^{-1} \prod_{m=i_{1}}^{i_{M-l}}\left(\sum_{i_{1}=1}^{N} \frac{\lambda_{i_{1}, m}^{-N_{r}}}{N_{r} !}\right)\right) \\
+\sum_{l=K+1}^{M} \sum_{\mathcal{D}_{l}}\left(\prod_{n=i_{1}}^{i_{N}} \lambda_{n}^{-1} \sum_{i_{1}, i_{2}, \cdots, i_{l-N+1} \in \mathcal{D}_{l}} \prod_{m=i_{1}}^{i_{l}} \lambda_{m}^{-N_{r}} \prod_{m=i_{1}}^{i_{M-l}}\left(\sum_{i_{1}=1}^{N} \frac{\lambda_{i_{1}, m}^{-N_{r}}}{N_{r} !}\right)\right) .
\end{gathered}
$$

TABLE III

Simulation PARAmeters

\begin{tabular}{c|lc}
\hline Notation & Parameter & Value \\
\hline$\alpha$ & Path-loss exponent & 3 \\
\hline $\mathcal{R}_{0}$ & Transmission rate & $\{1,2\}$ \\
\hline$N$ & Number of sources & $\{2,3,4\}$ \\
\hline$M$ & Number of relays & $\{3,4,5,6\}$ \\
\hline$K$ & Number of selected relays & $\{1,2,3,4\}$ \\
\hline$N_{r}$ & Number of antennas at each relay & $\{1,2,3,4,5\}$ \\
\hline$N_{d}$ & Number of antennas at destination & $\{1,2,3,4,5\}$ \\
\hline
\end{tabular}

Special Case 2. The derived analytical expressions are the generalized versions of i.i.d Rayleigh fading channels when $\bar{\gamma}_{n}=\bar{\gamma}_{n, m}=\bar{\gamma}_{m},(\forall n, m)[1]$.

\section{NUMERICAL RESUlTS AND DISCUSSIONS}

Herein, we present numerical results to illustrate the outage performance of RS Strategy $\mathcal{A}$ and Strategy $\mathcal{B}$. Insightful discussions related to the impact of different system parameters on the system performance will be presented. Monte-Carlo simulation results are further provided to support our analytical results. Table III shows the simulation parameters.

\section{A. i.i.d. Fading Channels}

1) Outage Performance of RS Strategy $\mathcal{A}$ and $\mathcal{B}$ : Here, we investigate the outage probability and diversity order of RS Strategy $\mathcal{A}$ and $\mathcal{B}$.

Fig. 3 illustrates the outage probability of RS Strategy $\mathcal{B}_{1}$ when $N=4, M=5, N_{r}=2, N_{d}=2$ and $K=1,2,3$, 4. The exact outage probability expressions are plotted along with the Monte-Carlo simulations. The analytical curves (54), (55) are in excellent agreement with Monte-Carlo simulations, confirming the accuracy of our derivations. Furthermore, the asymptotic lines perfectly predict the diversity orders and coding gains. It is observed that $K=1,2$ achieve the diversity order of 4 and 6 , respectively, indicating that the diversity order is determined by $(K+1) N_{d}(65)$, when $K<$ $\frac{(M+1) N_{r}}{N_{r}+N_{d}}=3$. We also observe that the maximum diversity order of $(K+1) N_{d}=8$ is achieved when $K=\frac{(M+1) N_{r}}{N_{r}+N_{d}}=3$. However, as $K$ increases form 3 to 4 the diversity decreases from 8 to $(M-K+1) N_{r}+N_{d}=6$ (67). Furthermore, although $K=4$ and $K=2$ both achieve the diversity order of $6, K=2$ outperforms $K=4$ for all SNR values. In conclusion, the performance improvement corresponding to the number of selected relays can only be obtained when the condition of $K \leq \frac{(M+1) N_{r}}{N_{r}+N_{d}}$ is satisfied. Otherwise, increasing $K$ significantly reduces the diversity order and coding gain.

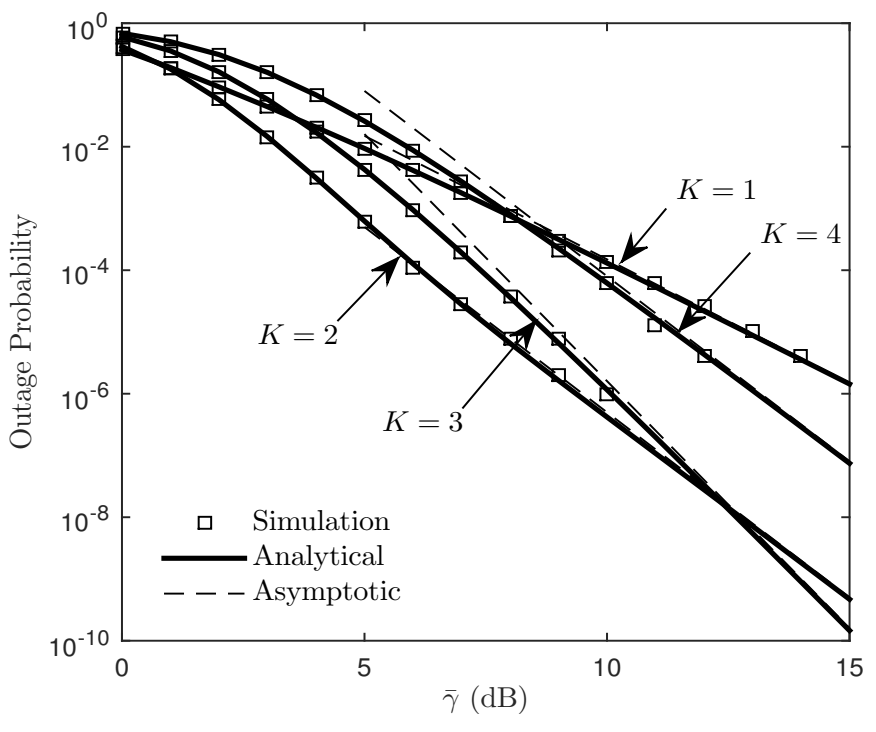

Fig. 3. Outage probability versus $\bar{\gamma}$ for Strategy $\mathcal{B}_{1}$ when $N=4, M=5$, $N_{r}=2, N_{d}=2$, and $K=1,2,3,4$.

In Fig. 4, we plot the outage probability of Strategy $\mathcal{A}$ and that of Strategy $\mathcal{B}_{2}$. We consider $N=4, M=6, K=2$, $N_{r}=1,2,5$ and $N_{d}=1,2,5$. This assumption satisfies the condition $N>K$. We observe that the outage performances of both strategies are exactly the same for the entire SNR regime. Furthermore, it can be seen that adding more antennas at relays does not improve outage performance in all SNRs. For example, for the case of $N_{r}>N_{d}$, the diversity order is always equal to $(K+1) N_{d}$. More precisely, the outage performance for $\left(N_{r}, N_{d}\right)=(5,1)$ is slightly better than that of $\left(N_{r}, N_{d}\right)=(2,1)$ in very low SNR regime. However, both curves have the same outage performance in medium to high SNR regime and their corresponding asymptotic slopes are identical and equal to $(K+1) N_{d}=3$. It can also be seen that when $\left(N_{r}, N_{d}\right)=(1,2)$, the outage performance gets better but still the slope of the curve at high SNR regime is determined by $(K+1) N_{d}$ and is equal to 6 . However, when $\left(N_{r}, N_{d}\right)=(1,5)$, the asymptotic slope of the curve is equal to $M N_{r}+N_{d}=11$. This indicates that asymptotic diversity of the system is determined by $(K+1) N_{d}$ and $M N_{r}+N_{d}$ for the case of $\frac{M}{K}>\frac{N_{d}}{N_{r}}$ and $\frac{M}{K}<\frac{N_{d}}{N_{r}}$, respectively (cf. Table I). Thus, the diversity improvement associated with the number of relays $M$ and the number of antennas at relays $N_{r}$ can only be obtained when the conditions $N_{d}>N_{r}$ and $\frac{M}{K}<\frac{N_{d}}{N_{r}}$ are satisfied. Otherwise, the gains corresponding to $M$ or $N_{r}$ are either negligible or even non-existent.

Fig. 5 depicts the outage probability of RS Strategy $\mathcal{A}\left(\mathcal{B}_{2}\right)$ 


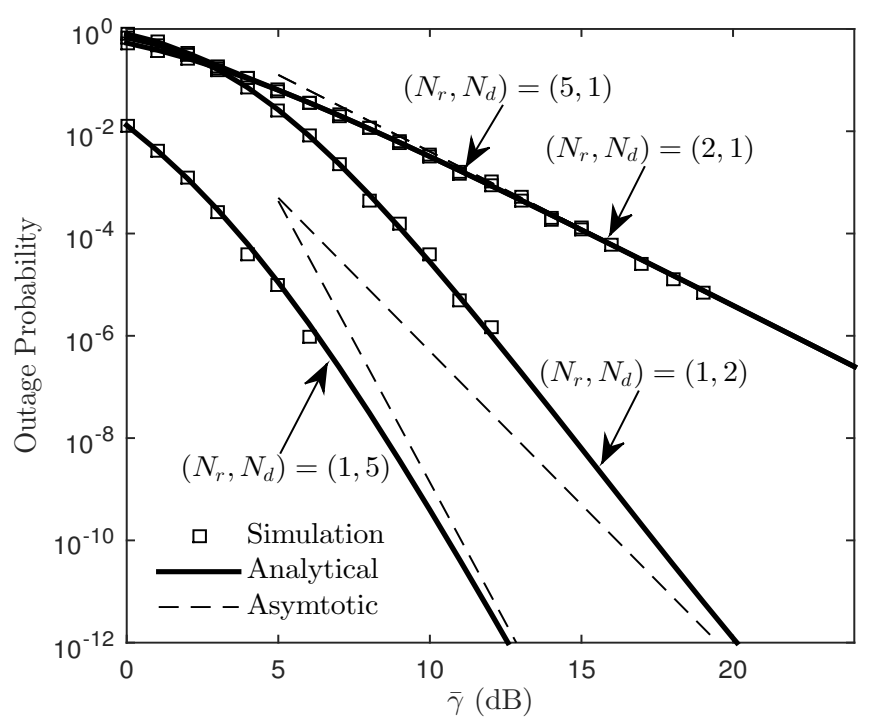

Fig. 4. Outage probability versus $\bar{\gamma}$ for RS Strategy $\mathcal{A}\left(\mathcal{B}_{2}\right)$ when $N=4$, $M=6, K=2, N_{r}=1,2,5$ and $N_{d}=1,2,5(N>K)$.

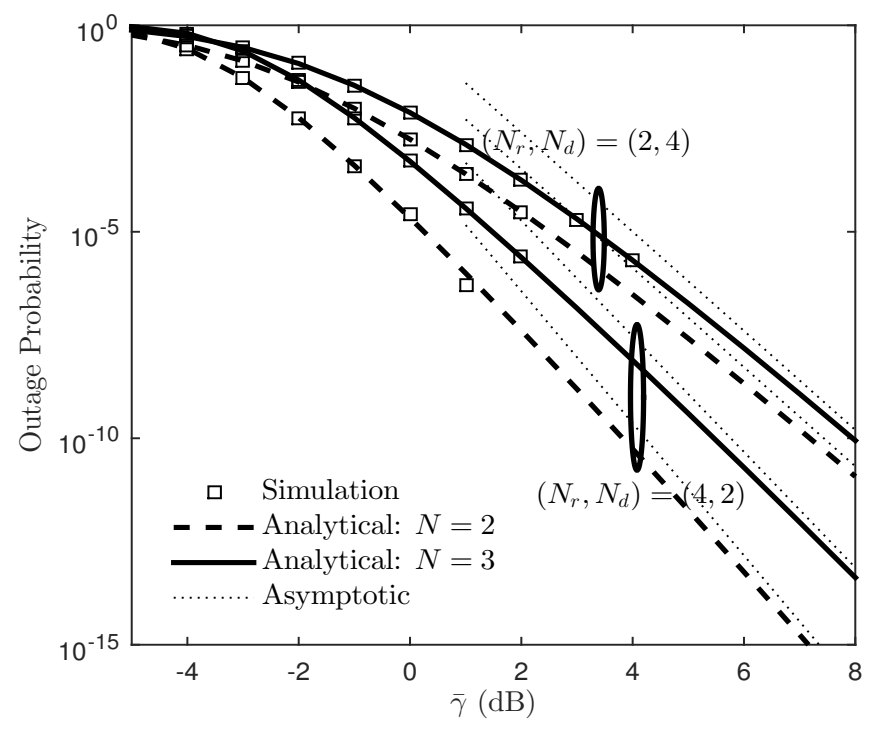

Fig. 5. Outage probability versus $\bar{\gamma}$ for RS Strategy $\mathcal{A}\left(\mathcal{B}_{2}\right)$ for $N=2,3$, $M=4, K=3, N_{r}=2,4$ and $N_{d}=2,4(N \leq K)$.

for $N=2,3, M=4, K=3, N_{r}=2,4$ and $N_{d}=2,4$. These assumptions satisfy the condition $N \leq K$. As expected, by increasing $N$ the system is more likely to undergo outage which results in higher outage values. It is also observed that when $N_{r}>N_{d}$, adding more sources also reduces the diversity order of the system from 16 to 14 as the diversity order is determined by $(M-N+1) N_{r}+N N_{d}$ (cf. Table I). While, for the case of $N_{r}<N_{d}$ the slope of the curves remain fix and is equal to $M N_{r}+N_{d}=12$.

Fig. 6 compares the outage performance between Strategy $\mathcal{A}\left(\mathcal{B}_{2}\right)$ and Strategy $\mathcal{B}_{1}$ when $N=3, M=6, K=2$, 3, $N_{r}=2$, and $N_{d}=2$. We observe that for $K=2$ $(N>K)$ Strategy $\mathcal{A}\left(\mathcal{B}_{2}\right)$ performs slightly better than Strategy $\mathcal{B}_{1}$ at low SNR values, while as SNR increases both have similar outage performances. This indicates that both strategies achieve the same diversity order and coding gain in high SNRs. However, for the case of $K=3,(N \leq K)$,

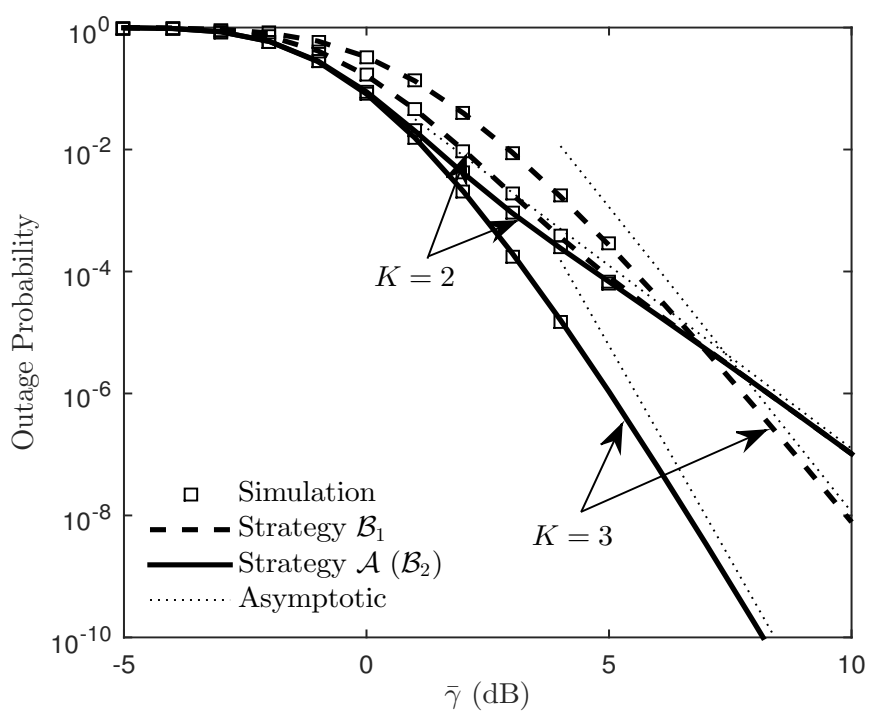

Fig. 6. Comparison between Strategy $\mathcal{A}\left(\mathcal{B}_{2}\right)$ and Strategy $\mathcal{B}_{1}$ when $N=3$, $M=6, K=2,3, N_{r}=2$, and $N_{d}=2$.

Strategy $\mathcal{A}\left(\mathcal{B}_{2}\right)$ achieves higher diversity order and thus significantly outperforms Strategy $\mathcal{B}_{1}$ in all SNRs. Specifically, to achieve a target outage rate of $10^{-6}, \mathrm{SNR}=5 \mathrm{~dB}$ is required for Strategy $\mathcal{A}\left(\mathcal{B}_{2}\right)$, while this increases to $8 \mathrm{~dB}$ for Strategy $\mathcal{B}_{1}$, indicating an SNR gain of $3 \mathrm{~dB}$. Thus, the achievable diversity order and coding gain of RS Strategy $\mathcal{B}_{1}$ are always equal or less than that of Strategy $\mathcal{A}$ or Strategy $\mathcal{B}_{2}$. Therefore, RS Strategy $\mathcal{B}_{1}$ does not provide any performance gain.

2) Performance Comparison With Two Benchmark Schemes: Here, we compare the outage performance of our proposed RS MIMO-NCC with two benchmark schemes, namely single-antenna RS NCC [17], [18], and RS MIMO-NCC with RAS at relays.

Fig. 7 plots the outage performance of single-antenna RS NCC [17], [18] and that of RS Strategy $\mathcal{A}\left(\mathcal{B}_{2}\right)$ when $N=3$, $M=5, N_{r}=2, N_{d}=2$, and $K=1,2,3$. As can be seen, RS MIMO-NCC achieves impressive performance gains. For example, the required SNR to achieve a target outage of $10^{-3}$ for single-antenna RS NCC is $17,11,10.5 \mathrm{~dB}$ for $K=1,2,3$, respectively. This reduces to 7 and $3 \mathrm{~dB}$ for RS MIMO-NCC with $K=1,2$. It further reduces to $2.5 \mathrm{~dB}$ for $K=3$. In addition, the asymptotic lines reveal that the diversity order of single-antenna RS NCC for $K=1,2$, and 3 are respectively equal to 2 and 3, and 6. On the other hand, RS MIMO-NCC achieves the diversity order of 4,6 , and 12 for $K=1,2,3$ (cf. Table I).

Fig. 8 compares the outage performance between the proposed RS MIMO-NCC with TAS and RAS at relays. We assume $N=2, M=4, N_{r}=2,3, N_{d}=2,3$ and $K=1,2$. It is observed that when $K=1$ or $K=2$ and $N_{d}>N_{r}$ TAS has slightly better outage performance in finite SNR regime. However, as SNR tends to infinity, the diversity order and coding gain for both schemes becomes identical, leading to the same outage performance. On the other hand, when $K=2$ and $N_{d}<N_{r}$, TAS achieves higher diversity order and thus significantly outperforms RAS in both finite and asymptotic SNRs. This indicates that the diversity gains 


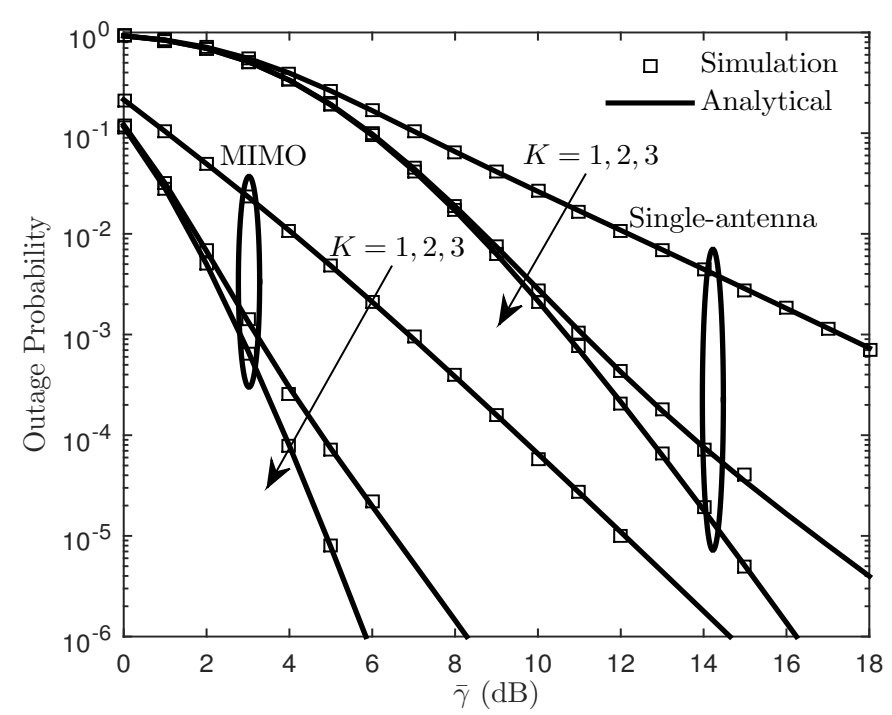

Fig. 7. Comparison between single-antenna RS NCC and RS Strategy $\mathcal{A}$ $\left(\mathcal{B}_{2}\right)$ when $N=3, M=5, N_{r}=2, N_{d}=2$, and $K=1,2,3$.

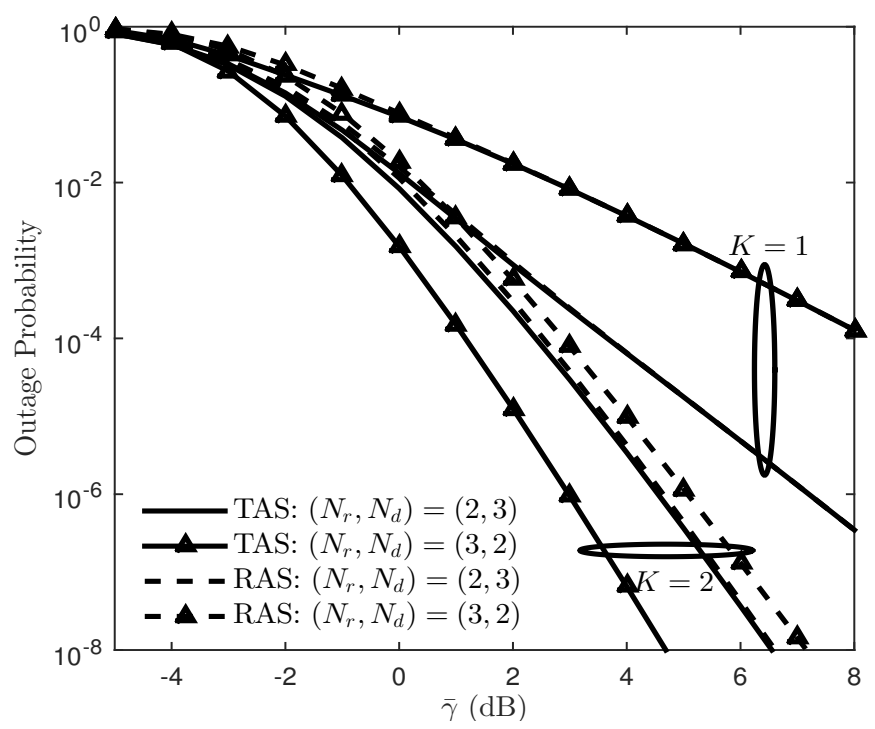

Fig. 8. Outage probability versus $\bar{\gamma}$ for Strategy $\mathcal{A}\left(\mathcal{B}_{2}\right)$ using TAS and RAS at relays. $N=2, M=4, N_{r}=2,3, N_{d}=2,3$, and $K=1,2$.

of TAS can only be obtained when the conditions $N \leq K$ and $N_{d}<N_{r}$ hold. Otherwise, RS MIMO-NCC with RAS at relays is more preferable due to the lower complexity.

\section{B. i.n.i.d. Fading Channels}

Here, we consider i.n.i.d. Rayleigh fading channels that take into account the effect of nodes' locations. In particular, we assume that the nodes are located in a 2-D plane where $d_{S_{n} R_{m}}$, $d_{S_{n} D}$, and $d_{R_{m} D}(\forall n, m)$ respectively denote the distances of source-to-relay, source-to-destination, and relay-to-destination links. The so-called "geometric gain" for $S_{n} \rightarrow R_{m}$ link with respect to $S_{1} \rightarrow D$ link can then be defined as ${ }^{5}$

$$
g_{n, m}=\left(\frac{d_{S_{n} R_{m}}}{d_{S_{1} D}}\right)^{\alpha}, \quad \forall n, m
$$

${ }^{5}$ We assume that $S_{1}$ is the most distant source to the destination.

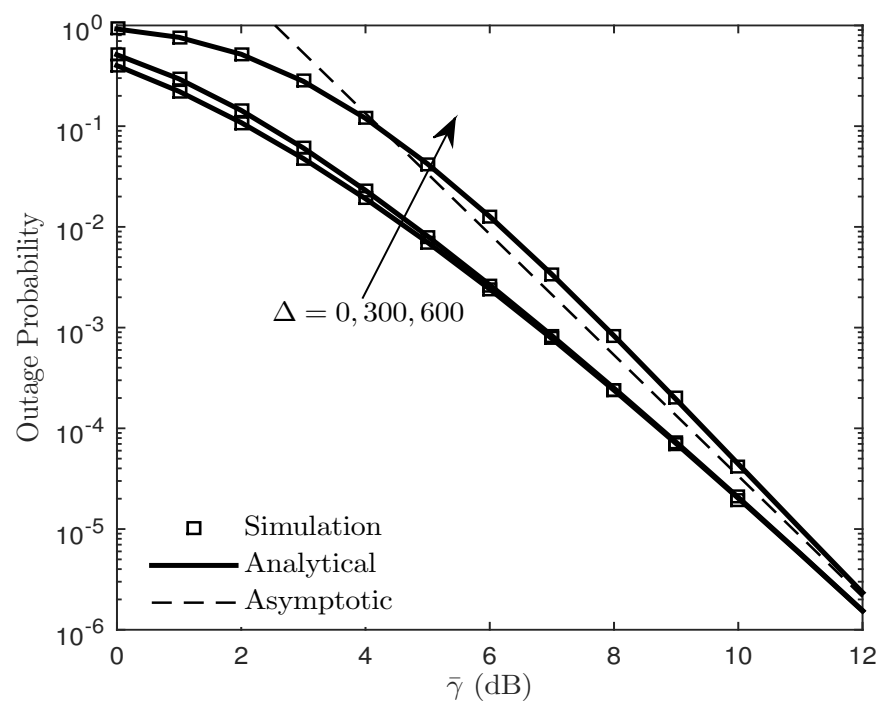

Fig. 9. Outage probability versus $\bar{\gamma}$ for RS Strategy $\mathcal{A}\left(\mathcal{B}_{2}\right)$ over i.n.i.d. fading channels when $N=3, M=3, K=2, N_{r}=2, N_{d}=2, \mathcal{R}_{0}=2$, and $\alpha=3$.

where $\alpha$ being the path-loss exponent.

Similarly, the geometric gain of $S_{n} \rightarrow D$ and $R_{m} \rightarrow D$ links with respect to $S_{1} \rightarrow D$ link are respectively given by

$$
g_{n}=\left(\frac{d_{S_{n} D}}{d_{S_{1} D}}\right)^{\alpha}, \quad \forall n
$$

and

$$
g_{m}=\left(\frac{d_{R_{m} D}}{d_{S_{1} D}}\right)^{\alpha}, \quad \forall m
$$

In Fig. 9, we plot the outage probability of RS MIMONCC over i.n.i.d. channels, assuming $N=3, M=3, K=2$, $N_{r}=2, N_{d}=2, \mathcal{R}_{0}=2$, and $\alpha=3$. The positions of $S_{1}, S_{2}, S_{3}$, and $D$ are kept fixed and are given by $\mathbf{X}_{S_{1}}=$ $\{0,300 \mathrm{~m}\}, \mathbf{X}_{S_{2}}=\{100 \mathrm{~m},-100 \mathrm{~m}\}, \mathbf{X}_{S_{3}}=\{0,-200 \mathrm{~m}\}$, $\mathbf{X}_{D}=\{1000 \mathrm{~m}, 0\}$. The positions of the relays, however, vary along the $x$ axis and are given by $\mathbf{X}_{R_{1}}=\{300+\Delta \mathrm{m}, 200 \mathrm{~m}\}$, $\mathbf{X}_{R_{2}}=\{200+\Delta \mathrm{m}, 0\}$, and $\mathbf{X}_{R_{3}}=\{300+\Delta \mathrm{m},-300 \mathrm{~m}\}$, where $\Delta \in\{0,300,600\}$ denotes the amount of the relay position shift. As can be seen the analytical results perfectly match simulations. In addition, the best outage performance occurs when the relays are in the vicinity of the sources i.e., $\Delta \approx 0$. However, as relays move towards the destination the outage performance deteriorates. We also observe that as SNR goes to infinity, the outage curves converge and the diversity for all curves is identical and equal to $(K+1) \tilde{N}=6$.

\section{CONCLUSION}

For NCC systems, we developed a new RS strategy that has the same performance as "max-min" criterion but does not need global CSI. To do this, we had to first analyze MIMONCC (which did not exist in the literature). In particular, we consider $N$ single-antenna sources, $M$ multiple-antenna relays and a single multiple-antenna destination. Considering the general case of i.n.i.d. fading channels, closed-form expressions for the outage probability, asymptotic outage probability, the diversity order, and the coding gain were derived and 
confirmed by Monte-Carlo simulations. In addition, we have shown that the diversity order of RS MIMO-NCC is unpredictable and highly dependent on the system configuration. In particular, our results interestingly demonstrated that increasing the number of relays and the number of antennas at relays does not necessarily improve the diversity order. Further, in contrast to single-antenna RS NCC system, increasing the number of sources may decrease the diversity order of the RS MIMO-NCC for some values of system parameters. Therefore, our analysis and results can benefit designing practical MIMONCC systems with RS.

Although our work provides valuable insights into the performance of RS MIMO-NCC systems, practical implementation issues such as channel estimation errors, cochannel interference, outdated CSI, and hardware costs could be investigated. This task is left as future work.

\section{REFERENCES}

[1] A. R. Heidarpour and M. Ardakani, "Diversity analysis of MIMO network coded cooperation systems with relay selection," in Proc. 2017 IEEE 86th Veh. Technol. Conf. (VTC-Fall), Sept. 2017, pp. 1-6.

[2] J. N. Laneman, D. N. C. Tse, and G. W. Wornell, "Cooperative diversity in wireless networks: Efficient protocols and outage behavior," IEEE Trans. Inf. Theory, vol. 50, no. 12, pp. 3062-3080, Dec. 2004.

[3] Y. Yang, H. Hu, J. Xu, and G. Mao, "Relay technologies for WiMax and LTE-advanced mobile systems," IEEE Commun. Mag., vol. 47, no. 10, pp. 100-105, Oct. 2009.

[4] R. Ahlswede, N. Cai, S. Y. R. Li, and R. W. Yeung, "Network information flow," IEEE Trans. Inf. Theory, vol. 46, no. 4, pp. 1204 1216, Jul. 2000.

[5] Y. Chen, S. Kishore, and J. Li, "Wireless diversity through network coding," in Proc. 2006 IEEE Wireless Commun. and Network. Conf., vol. 3, 2006, pp. 1681-1686.

[6] M. Yu, J. Li, and R. S. Blum, "User cooperation through network coding," in Proc. 2007 IEEE Inter. Conf. Commun., 2007, pp. 40644069.

[7] Z. Ding and K. K. Leung, "On the combination of cooperative diversity and network coding for wireless uplink transmissions," IEEE Trans. Veh. Technol., vol. 60, no. 4, pp. 1590-1601, May 2011.

[8] M. Xiao, J. Kliewer, and M. Skoglund, "Design of network codes for multiple-user multiple-relay wireless networks," IEEE Trans. Commun., vol. 60, no. 12 , pp. 3755-3766, Dec. 2012.

[9] H. Topakkaya and Z. Wang, "Wireless network code design and performance analysis using diversity-multiplexing tradeoff," IEEE Trans. Commun., vol. 59, no. 2, pp. 488-496, Feb. 2011.

[10] M. Xiao and M. Skoglund, "Multiple-user cooperative communications based on linear network coding," IEEE Trans. Commun., vol. 58, no. 12, pp. 3345-3351, Dec. 2010.
[11] A. R. Heidarpour, G. K. Kurt, and M. Uysal, "Finite-SNR DMT analysis for multisource multirelay NCC systems with imperfect CSI," in Proc. 2016 IEEE 84th Veh. Technol. Conf. (VTC-Fall), Sept. 2016, pp. 1-5.

[12] M. D. Renzo, M. Iezzi, and F. Graziosi, "On diversity order and coding gain of multisource multirelay cooperative wireless networks with binary network coding," IEEE Trans. Veh. Technol., vol. 62, no. 3, pp. 11381157, Mar. 2013

[13] A. R. Heidarpour, G. K. Kurt, and M. Uysal, "Finite-SNR diversitymultiplexing tradeoff for network coded cooperative OFDMA systems," IEEE Trans. Wireless Commun., vol. 16, no. 3, pp. 1385-1396, Mar. 2017.

[14] Z. C. Pereira, T. H. Ton, J. L. Rebelatto, R. D. Souza, and B. F. UchaFilho, "Generalized network-coded cooperation in OFDMA communications," IEEE Access, vol. 6, pp. 6550-6559, 2018.

[15] M. D. Renzo, M. Iezzi, and F. Graziosi, "Error performance and diversity analysis of multi-source multi-relay wireless networks with binary network coding and cooperative MRC," IEEE Trans. Wireless Commun., vol. 12, no. 6, pp. 2883-2903, 2013.

[16] C. Peng, Q. Zhang, M. Zhao, Y. Yao, and W. Jia, "On the performance analysis of network-coded cooperation in wireless networks," IEEE Trans. Wireless Commun., vol. 7, no. 8, pp. 3090-3097, Aug. 2008.

[17] M. D. Renzo, "On the achievable diversity of repetition-based and relay selection network-coded cooperation," IEEE Trans. Commun., vol. 62, no. 7, pp. 2296-2313, Jul. 2014.

[18] T. X. Vu, P. Duhamel, and M. D. Renzo, "On the diversity of networkcoded cooperation with decode-and-forward relay selection," IEEE Trans. Wireless Commun., vol. 14, no. 8, pp. 4369-4378, Aug. 2015.

[19] A. R. Heidarpour, M. Ardakani, and C. Tellambura, "Generalized relay selection for network-coded cooperation systems," IEEE Comm. Lett., vol. 21, no. 12, pp. 2742-2745, Dec. 2017.

[20] A. Bletsas, A. Khisti, D. P. Reed, and A. Lippman, "A simple cooperative diversity method based on network path selection," IEEE J. Sel. Areas Commun., vol. 24, no. 3, pp. 659-672, Mar. 2006.

[21] G. Amarasuriya, C. Tellambura, and M. Ardakani, "Two-way amplifyand-forward multiple-input multiple-output relay networks with antenna selection," IEEE J. Sel. Areas Commun., vol. 30, no. 8, pp. 1513-1529, Sept. 2012.

[22] A. Adinoyi and H. Yanikomeroglu, "Cooperative relaying in multiantenna fixed relay networks," IEEE Trans. Wireless Commun., vol. 6 , no. 2, pp. 533-544, Feb. 2007.

[23] G. Amarasuriya, C. Tellambura, and M. Ardakani, "Performance analysis framework for transmit antenna selection strategies of cooperative MIMO AF relay networks," IEEE Transactions on Vehicular Technology, vol. 60, no. 7, pp. 3030-3044, Sept. 2011.

[24] H. A. Suraweera, G. K. Karagiannidis, Y. Li, H. K. Garg, A. Nallanathan, and B. Vucetic, "Amplify-and-forward relay transmission with end-toend antenna selection," in Proc. 2010 IEEE Wireless Commun. and Network. Conf., Apr. 2010, pp. 1-6.

[25] Y. Kim and H. Liu, "Infrastructure relay transmission with cooperative MIMO," IEEE Trans. Veh. Technol., vol. 57, no. 4, pp. 2180-2188, Jul. 2008.

[26] S. Thoen, L. V. der Perre, B. Gyselinckx, and M. Engels, "Performance analysis of combined transmit-SC/receive-MRC," IEEE Trans. Commun., vol. 49, no. 1, pp. 5-8, Jan. 2001. 